\title{
Article \\ Navigating through the Lipid Metabolism Maze: Diagnosis and Prognosis Metabolites of Hepatocellular Carcinoma versus Compensated Cirrhosis
}

\author{
Iuliana Nenu 1,2,*, Horia Stefanescu 2,*(D), Bogdan Procopet ${ }^{1,2}$, Zeno Sparchez 1,2, Iulia Minciuna 1,2, \\ Tudor Mocan ${ }^{1,2}$ D , Daniel Leucuta ${ }^{3}$, Corina Morar ${ }^{4}$, Mircea Grigorescu ${ }^{1,2}$, Gabriela Adriana Filip 5 \\ and Carmen Socaciu 4 (iD
}

1 3rd Medical Department, "Iuliu Hatieganu" University of Medicine and Pharmacy, 400162 Cluj-Napoca, Romania; procopet.bogdan@umfcluj.ro (B.P.); sparchez.zeno@umfcluj.ro (Z.S.); minciuna.iulia@elearn.umfcluj.ro (I.M.); mocan.tudor@umfcluj.ro (T.M.); grigorescu.mircea@umfcluj.ro (M.G.)

2 Regional Institute of Gastroenterology and Hepatology, 400162 Cluj-Napoca, Romania

3 Department of Medical Statistics, "Iuliu Hatieganu" University of Medicine and Pharmacy, 400349 Cluj-Napoca, Romania; leucuta.daniel@umfcluj.ro

4 Center for Applied Biotechnology BIODIATECH, SC Proplanta, 400478 Cluj-Napoca, Romania; corina.hebristean@yahoo.co.uk (C.M.); csocaciudac@gmail.com (C.S.)

5 Department of Physiology, "Iuliu Hatieganu” University of Medicine and Pharmacy, 400006 Cluj-Napoca, Romania; gabriela.filip@umfcluj.ro

* Correspondence: iuliana.nenu@gmail.com (I.N.); horia.stefanescu@irgh.ro (H.S.)

Citation: Nenu, I.; Stefanescu, H.; Procopet, B.; Sparchez, Z.; Minciuna, I.; Mocan, T.; Leucuta, D.; Morar, C.; Grigorescu, M.; Filip, G.A.; et al. Navigating through the Lipid Metabolism Maze: Diagnosis and Prognosis Metabolites of Hepatocellular Carcinoma versus Compensated Cirrhosis. J. Clin. Med. 2022, 11, 1292. https://doi.org/ $10.3390 /$ jcm 11051292

Academic Editor: Jiangao Fan

Received: 31 January 2022

Accepted: 24 February 2022

Published: 26 February 2022

Publisher's Note: MDPI stays neutral with regard to jurisdictional claims in published maps and institutional affiliations.

Copyright: (C) 2022 by the authors. Licensee MDPI, Basel, Switzerland. This article is an open access article distributed under the terms and conditions of the Creative Commons Attribution (CC BY) license (https:// creativecommons.org/licenses/by/ $4.0 /)$.

\begin{abstract}
Background: The pursuit of finding biomarkers for the diagnosis and prognosis of hepatocellular carcinoma (HCC) has never been so paramount in the days of personalized medicine. The main objective of our study is to identify new biomarkers for diagnosing HCC, and to identify which patients are at risk of developing tumor recurrence, decompensation, or even possesses the risk of cancer-related death. (2) Methods: We have conducted an untargeted metabolomics study from the serum of 69 European patients-32 compensated cirrhotic patients without HCC (controls), and 37 cirrhotic patients with HCC with compensated underlying liver disease (cases), that underwent curative treatment (surgery or ablation), performing ultra-high-performance liquid chromatography coupled with electrospray ionization quadrupole time-of-flight mass spectrometry (UHPLC-QTOF- (ESI+)MS) with an emphasis on lipid metabolites. (3) Results: 1,25-dihydroxy cholesterol $(\mathrm{m} / \mathrm{z}=419.281)$, myristyl palmitate $(\mathrm{m} / \mathrm{z}=453.165), 25$-hydroxy vitamin $\mathrm{D} 2(\mathrm{~m} / \mathrm{z}=413.265), 12$-ketodeoxycholic acid $(\mathrm{m} / \mathrm{z}=391.283)$, lysoPC (21:4) $(\mathrm{m} / \mathrm{z}=558.291)$, and lysoPE (22:2) (m/z = 534.286) represent notable biomarkers that differentiate compensated cirrhosis from early HCC, and ceramide species are depleted in the serum of HCC patients. Regarding prognosis, no metabolite identified in our study could determine tumor relapse. To distinguish between the HCC patients that survived curative treatment and those at risk that developed tumor burden, we have identified two notable phosphocholines (PC (30:2); PC (30:1)) with AUROCs of 0.820 and 0.807, respectively, that seem to increase when patients are at risk. In a univariate analysis, arachidonic acid was the only metabolite to predict decompensation ( $\mathrm{OR}=0.1,95 \% \mathrm{CI}: 0-0.16, p<0.005)$, while in the multivariate analysis, dismally, no variable was associated with decompensation. Furthermore, in the multivariate analysis, we have found out for the first time that the increased expression of 1,25-dihydroxy cholesterol, myristyl palmitate, 12-keto deoxycholic acid, lysoPC (21:4), and lysoPE (22:2) are independent markers of survival. (4) Conclusions: Our study reveals that lipids play a crucial role in discriminating compensated cirrhosis and early hepatocellular carcinoma, and might represent markers of survival and prognosis in personalized and minimally invasive medicine.
\end{abstract}

Keywords: hepatocellular carcinoma; cirrhosis; lipid metabolites; biomarkers 


\section{Introduction}

One of the challenges that hepatologists face nowadays is the troublesome occurrence of hepatocellular carcinoma (HCC), the sixth most common cause of cancer worldwide, with an increasing incidence. Moreover, the World Health Organization estimates that more than a million people will die from liver cancer in 2030 [1,2]. In addition, due to late or limiting diagnosis methods, HCC has become more and more frequent on the doctor's agenda, often diagnosed in advanced stages, with a median overall survival time of 6-8 months [3].

Although $\alpha$-fetoprotein (AFP) is the most often used screening biomarker in clinical practice for early HCC detection, predicting tumor recurrence, and monitoring the oncological treatment, its poor sensitivity and specificity make it an unsatisfactory marker [4]. For this reason, new biomarkers are needed to identify among cirrhotic patients those at risk of developing liver malignancy, or to monitor treatment efficacy in the case of oncological patients. Along these lines, the concept of liquid biopsy has appeared in oncology for a minimally invasive positive diagnosis, prognosis, and overall disease monitoring. The term liquid biopsy can apply to cancer by-products, including circulating tumor cells (CTC), cell-free DNA (cfDNA), cell-free RNA (cfRNA), microRNA (miRNA), and extracellular vesicles (EVs), and last but not least, to metabolomics as well [5]. Because no organ other than the liver encompasses a myriad of lipids and other metabolites that are constantly interchanging in many biochemical reactions, metabolomics, which represents a compressive fingerprint of cell metabolism, also plays a crucial role in pursuing new diagnostic biomarkers and in identifying biochemical networks involved in prognosis and treatment monitoring [6,7].

Metabolic reprogramming is a hallmark characteristic of all cancer cells [8]. The most well-studied metabolic peculiarity is the Warburg effect, in which cancer cells use aerobic glycolysis instead of mitochondrial oxidative phosphorylation, leading to increased lactate production with a consequent role in cellular proliferation $[9,10]$. Because the liver is the primary metabolic hub of lipid metabolism through lipid homeostasis and energy balance maintenance, it is plausible to assume that many biochemical pathways involving lipids are disrupted [11]. Indeed, increased lipogenesis and lipolysis promote cancer cell growth, proliferation, and survival [11]. Moreover, fatty acid metabolism is enhanced, and phosphatidyl-choline (PC) and lyso-phosphatidylcholine (LPC) increase, while phosphatidyl-ethanolamine, -serine (PS) and -inositol, and ceramides are markedly reduced in HCC [11,12]. Other lipid metabolites play a double-edged sword role. Sphingolipid metabolism is quite controversial because ceramides induce a proapoptotic effect, whereas their phosphorylated compounds are involved in cancer growth, survival, and motility [13]. There are limited data regarding the involvement of ceramides and sphingosine-1 phosphate (S1P) as significant regulators of hepatocarcinogenesis, and their possible roles as biomarkers in HCC [14]. Some authors report a highly significant upregulation of long and very-long-chain ceramides (C16-C24) in the serum of patients with HCC compared to patients with cirrhosis, while other authors find a ceramide depletion in the serum of HCC patients $[15,16]$. Bile acids also have a dual role, with both protective and pathogenic roles being a matter of debate in drug-induced liver injury, NAFLD, and colon and liver cancers [17].

The main objective of our study is to identify new serum biomarkers, mainly lipids, and to define prognosis markers of early hepatocellular carcinoma, based on ultra-highperformance liquid chromatography coupled with electrospray ionization quadrupole time-of-flight mass spectrometry (UHPLC-QTOF- (ESI+)-MS). The present study identifies which patients are at risk of developing tumor recurrence, decompensation, and increased mortality. 


\section{Patients and Methods}

\subsection{Patient Characteristics and Sample Collection}

We have conducted a retrospective analysis of a prospectively collected database. The patients were admitted during 2016-2017 at the "Prof. Octavian Fodor" Regional Institute of Gastroenterology and Hepatology Cluj-Napoca, Romania, as follows: 37 patients with HCC (BCLC stages 0, A, and B) and 32 with compensated cirrhosis were admitted, with a period of three years of follow-up. The diagnosis of HCC was established based on the EASL guidelines for HCC using imaging and/or histopathology, and the stadialisation was performed according to the Barcelona clinic liver cancer (BCLC) score. All patients with compensated cirrhosis, representing the control group, had hepatitis $C$ virus etiology and were referred to interferon-free treatment. The diagnosis of liver cirrhosis was made using non-invasive methods such as abdominal ultrasound, transient elastography, serology, and liver biopsy in certain situations. HCC confirmation included two dynamic imaging examinations according to the EASL guidelines. Clinical characteristics included age, gender, and the etiology of the underlying liver disease. Liver function was determined using the Child-Pugh score, the model for end-stage liver disease (MELD) score, the alphafetoprotein (AFP), aspartate aminotransferase (AST), and alanine aminotransferase (ALT) levels, the platelet count, and the gamma-glutamyl transpeptidase (GGT) level.

Hepatic venous pressure gradient (HVPG) was performed to determine the portal hypertension grade, where clinically significant portal hypertension was defined over $10 \mathrm{mmHg}$ [18]. Tumor size and tumor number were assessed via imagistic tools at the time of inclusion, and, for HCC patients, the BCLC stage was determined. Contrast-enhanced CT or contrast-enhanced MRI were performed every three months during the first year and at least every 4-6 months thereafter to monitor disease progression. Independent physicians performed all follow-up examinations without prior knowledge of the study. The development of decompensation events was noted in the HCC group and was defined by the appearance of jaundice, ascites, hepatorenal syndrome, hepatic encephalopathy, and/or variceal bleeding. In the control group, patients with liver nodules or those with a history of decompensation or current symptoms of such were excluded. Patients with HCC developed on the non-cirrhotic liver, patients with advanced HCC (BCLC classes C and D), and those with concomitant cancer other than HCC were also excluded from the study group. Pregnant patients, as well as patients younger than 18 years old were excluded. The patients were followed up until November 2020. The study was performed according to the Declaration of Helsinki, and both groups signed the informed consent of the examination approved by the local ethical guidelines for storing biological samples. The cohort was prospectively studied, and serum lipid parameters were analyzed in retained serum samples, stored previously at $-80^{\circ} \mathrm{C}$.

\subsection{Metabolomic Analysis}

\subsubsection{Sample Preparation}

The patients included in this study had blood collected by venipuncture in sterile vacutainers anticoagulant, and samples were kept at $-80^{\circ} \mathrm{C}$. They were labeled using confidential numerical codes. A volume of $0.6 \mathrm{~mL}$ methanol (99\%) was added for each volume of $0.2 \mathrm{~mL}$ of plasma, and the mixture was vortexed to precipitate proteins for $30 \mathrm{~s}$. Afterward, the composition was kept for $5 \mathrm{~min}$ in an ultrasonication instrument, followed by $5 \mathrm{~min}$ at $-20^{\circ} \mathrm{C}$. The supernatant was collected following centrifugation at 10,000 rpm for $10 \mathrm{~min}\left(4^{\circ} \mathrm{C}\right)$.

Afterward, probes were subjected to ultrasound in a water bath for $5 \mathrm{~min}$ and left at $-20{ }^{\circ} \mathrm{C}$ to increase protein precipitation. The samples were centrifuged at $5000 \mathrm{rpm}$ for $10 \mathrm{~min}$ to separate the protein supernatant. The supernatant was collected and filtered using nylon filters $(0.2 \mu \mathrm{m})$. The samples were placed in vials, and then in an autosampler to be injected into the UHPLC MS system. 


\subsubsection{UHPLC-QTOF-ESI+-MS Analysis}

Plasma metabolomic profiling was performed using ultra-high-performance liquid chromatography coupled with electrospray ionization-quadrupole-time of flight-mass spectrometry (UHPLC-QTOF-ESI+-MS) in a ThermoFisher Scientific UHPLC Ultimate 3000 instrument equipped with a quaternary pump, a Dionex delivery system, and MS detection equipment with MaXis Impact (Bruker Daltonics). The metabolites were separated using a Thermo Scientific Acclaim C18 column $(3 \mu \mathrm{m}, 2.1 \times 50 \mathrm{~mm}$, pore size $30 \mathrm{~nm})$ at $40{ }^{\circ} \mathrm{C}$. The mobile phase consisted of $0.1 \%$ formic acid in water (A) and $0.1 \%$ formic acid in acetonitrile (B). The flow rate was set at $0.5 \mathrm{~mL} \mathrm{~min}^{-1}$. The gradient was: 5 to $15 \% \mathrm{~A}$ (0-3 $\mathrm{min}), 15-50 \%$ A (3-6 min), 50-95\% (6-9 min), isocratic until $15 \mathrm{~min}$, and afterwards decreased from 95 to $5 \%(15-20 \mathrm{~min})$. The elution time was set for $20 \mathrm{~min}$. The volume of the injected extract was $5 \mu \mathrm{L}$, the column temperature was $40^{\circ} \mathrm{C}$. Several QC samples obtained from each group were used in parallel to calibrate the separations.

Metabolite identification was performed using specific MS parameters: ionization mode ESI+ positive, MS calibration with sodium formate, capillary voltage $3500 \mathrm{~V}$, pressure for the nebulizing gas 2.8 bar, drying gas flow $12 \mathrm{l} / \mathrm{min}$, drying temperature $300^{\circ} \mathrm{C}$. The control of the instrument and the data processing were conducted using the specific software TofControl 3.2, HyStar 3.2, Data Analysis 4.2 (Bruker, Daltonics), and Chromeleon, respectively.

\subsection{Data Processing and Statistical Analysis}

The base peak chromatograms and all MS spectra were first processed by Compass DataAnalysis 4.2 (Bruker Daltonics, GmbH, Bremen, Germany), using the find molecular feature (FMF) algorithm, and matrix generation was achieved using Profile Analysis 2.1 (Bruker Daltonics, GmbH, Bremen, Germany). The time alignment, spectral background extraction, MS recalibration, normalization by the sum of the bucket values in analysis, and an $80 \%$ bucket filter were the chosen parameters.

MetaboAnalyst v5.0 online software was used for univariate and multivariate analyses. The matrices representing the peak intensity versus $\mathrm{m} / \mathrm{z}$ values for each sample and subgroups of samples were tested using the most relevant statistical parameters to reflect the discrimination between groups, the prediction, and the correlation maps. Therefore, the comparative statistical approaches selected were the volcano test, the variable importance in the projection (VIP) values, the scores and loadings plots of principal component analysis (PCA) and partial least square discriminant analysis (PLSDA) including cross-validation parameters, the random forest-based prediction, and the calculation of $p$-values by $t$-test via ANOVA. The same software was applied to build the receiver operating characteristic (ROC) curves and area values under ROC curves (AUC) to evaluate the potential biomarkers' sensibility and selectivity. Finally, enrichment analysis and pathway matches were established to find the significant metabolites for altered pathways. The relevant molecules, according to statistical analysis, were identified by searches on specialized databases, such as Lipid Maps (http:/ /www.lipidmaps.org (accessed on 20 December 2021)) and the Human Metabolome Database (http:/ /www.hmdb.ca (accessed on 20 December 2021)), and PubChem (https://pubchem.ncbi.nlm.nih.gov/ (accessed on 20 December 2021). The PubChem database codes of each identified metabolite were included.

\subsection{Data Processing and Statistical Analysis for Prognosis}

Regarding prognosis, the overall survival was defined as the time from treatment until death or the study ending date (November 2020). Disease-free survival was defined as the time from treatment until recurrence or the study's end date (November 2020). Further, known predictors for survival were added as adjusting variables in multivariate Cox regression models. The proportional hazard assumption was checked with a formal statistical test for all models, while the linear functional form for continuous variables was checked using model residuals plots inspection. For multivariate models, multicollinearity was checked with variance inflation factors. The two-tailed $p$-value was computed for all statistical tests, and the results were statistically significant for values below 0.05 . Data were 
analyzed using the R environment for statistical computing and graphics ( $\mathrm{R}$ Foundation for Statistical Computing, Vienna, Austria), version 3.6.3 [R Core Team. R: A Language and Environment for Statistical Computing and IBM SPSS Statistics 25.0.] (IBM, Armonk, NY, USA).

\section{Results}

\subsection{Patient Characteristics}

The present study included patients with HCC (group HCC) and patients with compensated cirrhosis as controls (group C). Because all HCV patients from the control group received interferon-free treatment, they were rigorously evaluated clinically and biochemically, and imaging (ultrasound $\pm \mathrm{CT}$ ) was performed. In effect, the national protocol in 2016 banned interferon-free therapy in patients who had cancers, including HCC and decompensated (or a history of decompensated) liver disease. Thus, all of our control patients had compensated hepatitis $C$ cirrhosis. Precisely for this reason, knowing with certainty that the patients from the control group did not have liver tumors, we compared patients with liver cirrhosis with those with HCC to determine different biomarkers of diagnosis and prognosis.

Most patients with HCC had compensated liver disease, with hepatitis $\mathrm{C}$ virus (HCV) representing the primary etiology $-43.24 \%$, followed by alcoholic cirrhosis (32.4\%), and chronic hepatitis B (HBV) infection (16.21\%). Only six patients had Child-Pugh B class 7 points with preserved liver function. All 37 HCC patients received curative treatment: $9(24.32 \%)$ underwent surgical resection and $28(75.67 \%)$ received percutaneous ultrasoundguided tumor ablation. The mean duration of follow-up was $29.23 \pm 10.74$ months. Within the observation time, 13 (35.13\%) patients died (group HCd), while 24 survived (group HCs). One patient from the cirrhotic control group died, but without liver-related causes. Regarding tumor relapse, 22 patients $(59.45 \%)$ were diagnosed with tumor recurrence.

Regarding BCLC class, the HCC patients are classified as follows: 4 BCLC 0, 15 BCLC $\mathrm{A}$, and 18 BCLC B with compensated underlying liver disease. BCLC-B class is characterized by extensive heterogeneity due to the wide range of liver function (Child-Pugh A or B cirrhosis) and variable lesion number and size. Bolondi et al. proposed a sub-classification of intermediate stage HCC so that BCLC B patients might benefit from other treatment options besides transarterial chemoembolization [19]. All 18 BCLC B patients from our study had undergone curative treatment. Of these, 16 correspond to "up to seven criteria" as follows: 9 patients had tumors less than $6.5 \mathrm{~cm}$ diameter and underwent surgery, 7 patients had two or three nodules, with a total sum diameter less than $8 \mathrm{~cm}$. The last 2 patients had four nodules, in which surgery and ablation were combined. Unfortunately, liver transplantation is not feasible in our center, so surgical resection or percutaneous ablation were also performed in patients who met the Milan criteria.

None of the patients with HCC and HCV received interferon-free treatment before diagnosis of liver tumors. One had a history of treatment with ribavirin and interferon with no virological response. Subsequently, after the curative treatment, out of the sixteen patients with HCC and HCV, eight underwent DAA treatment with sofosbuvir/ledipasvir. Of these, two had recurrence. Of the six patients with HCC and HBV, one was on lamivudine therapy. Subsequently, none of these were treated with entecavir after curative treatment. All the patients included in this study, including those with HCC and ethanolic cirrhosis, were abstinent.

The baseline characteristics of the study population are depicted in Table 1.

\subsection{Univariate and Multivariate Analysis of Metabolic Profile}

\subsubsection{Analysis of Raw Data Based on UHPLC-QTOF-ESI+-MS Peak Intensities}

The data released from the UHPLC-QTOF-ESI+-MS analysis were included in matrices representing the $\mathrm{m} / \mathrm{z}$ values and peak intensities for each of the more than 300 molecules separated. After eliminating small signals with $\mathrm{S} / \mathrm{N}$ values $<10$ and molecules with peak intensities less than 25,000, the number of peaks remained at around 250. Only metabolites 
detected in more than $80 \%$ of the samples $(n=154)$ were subsequently included in the statistical analysis. The aligned matrix was converted to a .csv file and processed using the online software Metaboanalyst 5.0.

Table 1. Baseline characteristics of the study population.

\begin{tabular}{|c|c|c|c|}
\hline & HCC (Group HC) & Cirrhosis (Group C) & $p$ Value \\
\hline \multicolumn{4}{|c|}{ General Data } \\
\hline Number & 37 & 32 & \\
\hline Age mean SD * & $64.62(6.29)$ & $55.97(8.1)$ & $<0.001$ \\
\hline \multicolumn{4}{|l|}{ Sex, no $(\%) * *$} \\
\hline Females & $10(27.03)$ & $20(62.5)$ & \\
\hline Males & 27 (72.97) & $11(37.5)$ & $<0.003$ \\
\hline \multicolumn{4}{|l|}{ Etiology $(\%)^{* *}$} \\
\hline $\mathrm{HCV}$ & $16(43.24)$ & $32(100 \%)$ & \\
\hline HVB & $6(16.21)$ & & \\
\hline Alcohol & $12(32.4)$ & & \\
\hline Cholestatic & $1(2.7)$ & & \\
\hline NAFLD & $2(5.4)$ & & $<0.01$ \\
\hline Dead (yes), no (\%) & $13(35.13)$ & $1(3.33)$ & $<0.001$ \\
\hline \multicolumn{4}{|c|}{ Laboratory values } \\
\hline $\mathrm{ALAT}^{*}$ & $37(21-59)$ & $74.5(46.25-108.25)$ & 0.015 \\
\hline ASAT * & $57(35-82)$ & $72.5(52.25-97.75)$ & 0.76 \\
\hline$\gamma \mathrm{GT}^{*}$ & $61(33.5-91.5)$ & $60(42-129)$ & 0.67 \\
\hline Total bilirubin * & $1(0.8-1.6)$ & $1.15(0.9-1.35)$ & 0.67 \\
\hline Creatinine * & $0.73(0.61-0.95)$ & $0.72(0.58-0.84)$ & 0.2 \\
\hline Albumin * & $3.8(3.6-4.1)$ & $4.1(4-4.3)$ & $<0.01$ \\
\hline Hemoglobin * & $14.2(12.2-15.2)$ & $14.5(13.7-15.8)$ & 0.06 \\
\hline PMN count * & $3.37(2.5-4.62)$ & $3.11(2.52-3.95)$ & 0.089 \\
\hline Platelet count* & $117(82-147)$ & $103(79-144.5)$ & 0.725 \\
\hline $\mathrm{Na}^{*}$ & $140(138-142)$ & 140 (139-143.25) & 0.3 \\
\hline $\mathrm{K}^{*}$ & $4.3(4.07-4.6)$ & $3.9(3.8-4.3)$ & 0.012 \\
\hline $\mathrm{AFP}^{*}$ & $10.1(5.5-58.8)$ & $13.5(8.33-19.62)$ & 0.016 \\
\hline \multicolumn{4}{|c|}{ The severity of Liver Disease } \\
\hline \multicolumn{4}{|l|}{ Child-Pugh ** } \\
\hline A & $31(74.1 \%)$ & $32(100 \%)$ & $<0.01$ \\
\hline B & $6(22.2 \%)$ & & \\
\hline \multicolumn{4}{|l|}{ MELD ** } \\
\hline$\leq 9$ & $16(43.24 \%)$ & $19(59.38 \%)$ & 0.23 \\
\hline \multirow[t]{2}{*}{$10-19$} & $1(2.7 \%)$ & $13(40.62 \%)$ & \\
\hline & $20(54.5 \%)$ & & \\
\hline \multicolumn{4}{|l|}{ Portal pressure * } \\
\hline HVPG & $15(11-18)$ & $14(11-16)$ & 0.448 \\
\hline \multicolumn{4}{|c|}{ HCC staging } \\
\hline \multicolumn{4}{|l|}{ BCLC ** } \\
\hline 0 & $4(11 \%)$ & & \\
\hline A & $15(40 \%)$ & & \\
\hline B & $18(49 \%)$ & & \\
\hline \multicolumn{4}{|l|}{ Tumor number ** } \\
\hline$<3$ & $34(91.89 \%)$ & & \\
\hline $3-5$ & $3(8.11 \%)$ & & \\
\hline$>5$ & 0 & & \\
\hline
\end{tabular}

Milan criteria **

$\begin{array}{ll}\text { In } & 19(51.35 \%) \\ \text { Out } & 18(48.65 \%)\end{array}$

* = values expressed as median IQR; ${ }^{* *}=$ values expressed as absolute value + per cents; AFP = alpha-feto protein $\mathrm{HCC}=$ hepatocellular carcinoma; $p=$ level of significance; $\mathrm{HCV}=$ hepatitis $\mathrm{C}$ virus; $\mathrm{HBV}=$ hepatitis $\mathrm{B}$ virus; NAFLD = non-alcoholic fatty liver disease; ALAT = alanine aminotransferase; ASAT = aspartate aminotransferase $\Gamma \mathrm{t}$ = gamma glutamyl transferase; $\mathrm{Na}=$ sodium; $\mathrm{K}=$ potassium; $\mathrm{MELD}=$ model for end stage liver disease; $\mathrm{HVPG}=$ hepatic venous pressure gradient; $\mathrm{BCLC}=$ Barcelona clinic liver cancer. 
Table S1 (Supplementary File) includes a list of molecules separated and identified $(n=154)$, and their average LC-MS peak intensities and standard deviations (SD). The $\mathrm{m} / \mathrm{z}$ values represent $[\mathrm{M}+1]$ where $M$ is the individual molecular mass. Table S2 (Supplementary File) includes a list of the same molecules and their average intensities for the subgroups HCd $(n=13)$ vs. HCs $(n=24)$.

The main classes of molecules are represented by fatty acid derivatives (31), glycerophospholipids and lysoderivates (15 and 27, respectively), diacyl- and monoacyl glycerols and phosphoglycerols (18, 6 , and 5 respectively), ceramides and sphingosine derivatives (18), sterols and bile acids (11), and acylcarnitines (11), as well amino acids, choline derivatives (9) and oxylipins as eicosanoid inflammatory mediators (4). Their relative levels in the HCC (HC group) vs. cirrhosis group (C), and their values in the HCd group vs. survivals of the HC group, are presented in Table 2.

Table 2. Metabolites separated and identified in different patient groups ( $\mathrm{HC}, \mathrm{C}, \mathrm{HCd}$, and $\mathrm{HCs}$ ) and the ratios of their levels in the groups HC vs. C, and HCd vs. HCs.

\begin{tabular}{|c|c|c|c|c|c|c|c|}
\hline $\mathbf{m} / \mathbf{z}$ & & $\mathrm{HC} / \mathrm{C}$ & $\mathrm{HCd} / \mathrm{HCs}$ & $\mathbf{m} / \mathbf{z}$ & & $\mathrm{HC} / \mathrm{C}$ & $\mathrm{HCd} / \mathrm{HCs}$ \\
\hline \multicolumn{4}{|c|}{ Steroids, Incl.Bile Acids } & \multicolumn{4}{|c|}{ Fatty Acid Derivatives } \\
\hline 419.281 & 1,25 DiOH cholesterol & 6.90 & 0.96 & 453.165 & Myristyl palmitate & 4.12 & 0.97 \\
\hline 391.283 & 12-Ketodeoxycholic acid & 3.24 & 0.89 & 313.255 & Icosanoic (arahidic) acid C20:0 & 2.25 & 0.93 \\
\hline 413.265 & 25-hydroxy vitamin D2 & 3.06 & 0.89 & 331.284 & Docosapentenoic acid (C22:5) & 2.07 & 0.95 \\
\hline 409.328 & Ursocholic acid & 1.38 & 0.9 & 303.181 & Eicosapentenoic acid (C20:5) & 1.85 & 0.86 \\
\hline 473.327 & 3-Sulfodeoxycholic acid & 1.36 & 0.91 & 425.130 & Lauryl palmitate & 1.83 & 1.09 \\
\hline 271.264 & Estrone & 1.17 & 1.13 & 325.250 & Methyl-7-eicosenoic acid & 1.79 & 0.92 \\
\hline 585.268 & Cholic acid glucuronide & 1.14 & 1.19 & 318.291 & N-methyl arachidonoyl amine & 1.76 & 1.07 \\
\hline 289.159 & Testosterone/DHEA & 1.14 & 1.02 & 397.268 & Hexacosanoic acid (C26:0) & 1.59 & 1.02 \\
\hline 421.338 & Dihomodeoxycholic acid & 0.60 & 0.92 & 427.390 & N-stearoyl arginine & 1.23 & 1.03 \\
\hline 369.295 & Testosterone sulfate/DHEAS & 0.55 & 0.92 & 326.354 & Oleoyl Ethanolamide & 1.17 & 1.03 \\
\hline 273.174 & Estradiol & 0.50 & 0.7 & 350.338 & $\begin{array}{c}\text { Dihomo-gamma-linolenoyl (C18:3) } \\
\text { Ethanolamine }\end{array}$ & 1.10 & 1.01 \\
\hline \multicolumn{4}{|c|}{ Oxylipins } & 299.126 & 2-hydroxy oleic acid (C18:1) & 1.06 & 1.04 \\
\hline 353.271 & Prostaglandin E2 & 2.54 & 0.93 & 338.341 & Docosenamide (C22) & 1.02 & 1.01 \\
\hline 267.266 & Tetranor 12-HETE & 1.15 & 0.96 & 324.325 & Linoleoyl ethanolamide & 1.01 & 0.95 \\
\hline 406.328 & 15-HETE-GABA & 0.49 & 0.77 & 341.298 & Docosanoic acid (C22:0) & 0.94 & 0.96 \\
\hline 383.207 & $\begin{array}{l}\text { 12-Oxo-trihydroxy-leukotriene } \\
\text { B4 }\end{array}$ & 0.22 & 1.06 & 348.319 & $\begin{array}{l}\text { O-Arachidonoyl }(\mathrm{C} 20: 4, \mathrm{n}-6) \\
\text { Ethanolamine }\end{array}$ & 0.86 & 1.06 \\
\hline \multicolumn{4}{|c|}{ Acyl carnitines } & 269.217 & Heptadecenoic acid (C17:1) & 0.65 & 1.03 \\
\hline 414.289 & Heptadecanoyl carnitine & 3.92 & 2.47 & 501.375 & Palmitoleyl linolenate & 0.55 & 0.93 \\
\hline 290.261 & Adipoyl carnitine & 2.81 & 1.09 & 301.142 & Eicosahexaenoic acid (C20:6) & 0.45 & 0.96 \\
\hline 316.317 & Decanoylcarnitine & 1.90 & 1.1 & 529.407 & Linoleyl linoleate & 0.43 & 1.04 \\
\hline 304.295 & Pimelyl carnitine & 1.78 & 1.03 & 285.282 & Stearic acid & 0.42 & 1.06 \\
\hline 288.266 & Octanoyl carnitine & 1.66 & 1.08 & 295.182 & 2-Hydroxy linolenic acid (C16:3) & 0.40 & 0.97 \\
\hline 230.24 & Butenyl carnitine & 1.28 & 1.01 & 279.160 & Linolenic acid (C18:3) & 0.33 & 0.98 \\
\hline 332.327 & 3-hydroxydecanoyl carnitine & 1.21 & 0.99 & 163.052 & Hydroxy adipic acid & 0.26 & 1.18 \\
\hline 312.326 & Decadienoyl carnitine & 1.18 & 1.01 & 249.189 & Hexadecatetraenoic acid C16:4 & 0.22 & 1.05 \\
\hline 374.259 & Dodecanedioylcarnitine & 1.15 & 0.93 & 229.131 & Myristic acid (C14) & 0.21 & 1.03 \\
\hline 388.355 & $\begin{array}{l}\text { 3-Hydroxytetradecanoyl } \\
\text { carnitine }\end{array}$ & 1.09 & 1.04 & 257.204 & Palmitic acid (C16:0) & 0.20 & 0.93 \\
\hline 310.308 & Decatrienoyl carnitine & 0.98 & 0.93 & 339.366 & Dimethyl-2-eicosenoic acid (C22) & 0.16 & 1.09 \\
\hline \multicolumn{4}{|c|}{ Aminoacid and choline derivatives } & 245.077 & Hydroxy myristic acid (C14) & 0.14 & 1.02 \\
\hline 258.265 & Glycerophosphocholine & 1.69 & 1.02 & 305.145 & Arahidonic acid (C20:4) & 2.83 & 1.51 \\
\hline
\end{tabular}


Table 2. Cont.

\begin{tabular}{|c|c|c|c|c|c|c|c|}
\hline $\mathrm{m} / \mathrm{z}$ & & $\mathrm{HC} / \mathrm{C}$ & $\mathrm{HCd} / \mathrm{HCs}$ & $\mathrm{m} / \mathrm{z}$ & & $\mathrm{HC} / \mathrm{C}$ & $\mathrm{HCd} / \mathrm{HCs}$ \\
\hline \multicolumn{4}{|c|}{ Steroids, Incl.Bile Acids } & \multicolumn{4}{|c|}{ Fatty Acid Derivatives } \\
\hline 166.073 & Phenyl alanine & 1.10 & 0.94 & 415.209 & Ascorbyl palmitate & 2.94 & 0.99 \\
\hline 183.082 & Phosphoryl choline & 0.46 & 0.98 & \multicolumn{4}{|c|}{ Sphingolipids } \\
\hline 161.1 & Tryptamine & 0.43 & 0.59 & 274.265 & C16-Sphingosine & 1.64 & 1.07 \\
\hline 149.024 & Methionine & 0.27 & 0.98 & 526.518 & Ceramide (d18:1/14:0 (2OH)) & 1.49 & 1.05 \\
\hline 158.15 & Tiglylglycine & 0.12 & 1.08 & 554.547 & Ceramide (d18:1/16:0 (2OH)) & 1.10 & 1.04 \\
\hline 171.14 & Glyceraldehyde 3-phosphate & 0.06 & 1.04 & 354.360 & C16 Sphinganine 1-P & 0.99 & 1.03 \\
\hline 364.346 & a-linolenyl choline (C18:3) & 0.63 & 0.96 & 623.245 & Ceramide (d18:1/22:0) & 0.88 & 1 \\
\hline 366.374 & a-linoleyl choline (C18:2) & 0.87 & 1.09 & 584.464 & Ceramide (d18:0/18:0 (2OH)) & 0.63 & 0.99 \\
\hline \multicolumn{4}{|c|}{ Lyso Phospholipids } & 582.576 & Ceramide (d18:1/18:0 (2OH)) & 0.61 & 1.08 \\
\hline 558.291 & LysoPC (21:4) & 2.83 & 1.07 & 628.495 & Ceramide (t18:0/20:0 (2OH)) & 0.58 & 0.98 \\
\hline 522.357 & LysoPC (18:1) & 2.11 & 1.06 & 540.441 & Ceramide (d18:0/16:0) & 0.58 & 1 \\
\hline 534.286 & LysoPE (22:2) & 2.08 & 1.09 & 672.525 & GlycoCeramide (d18:1/14:0) & 0.58 & 0.93 \\
\hline 437.191 & LysoPA (18:1/0:0) & 1.89 & 0.99 & 703.574 & Sphingomyelin 18:2/16:0 & 0.50 & 1 \\
\hline 524.37 & LysoPC (18:0) & 1.68 & 1.02 & 496.419 & Ceramide (d15:1/16:0) & 0.49 & 0.97 \\
\hline 482.338 & LysoPE (18:0) & 1.54 & 1.25 & 596.512 & Ceramide (d20:0/18:0) & 0.48 & 1.28 \\
\hline 483.121 & LysoPA (22:6/0:0) & 1.51 & 1.23 & 508.459 & Ceramide (d18:2/14:0) \& isom. & 0.47 & 1.05 \\
\hline 510.372 & LysoPE (20:0) & 1.49 & 1.21 & 568.469 & Ceramide (d18:0/18:0) & 0.45 & 1.23 \\
\hline 480.332 & LysoPE (18:1) & 1.42 & 1.18 & 524.448 & $\begin{array}{l}\text { Ceramide (d14:1/18:1 (2OH)) } \\
\text { isomizom }\end{array}$ & 0.39 & 1.31 \\
\hline 520.342 & LysoPC (18:2) & 1.41 & 1.16 & 612.503 & Ceramide (d18:0/20:0 (2OH)) & 0.36 & 1.08 \\
\hline 495.297 & LysoPA (22:0/0:0) & 1.38 & 0.97 & \multicolumn{4}{|c|}{ Monoacyl glycerols } \\
\hline 546.354 & LysoPC (20:3) & 1.35 & 1.06 & 379.263 & MG (20:4/0:0/0:0) & 1.45 & 0.83 \\
\hline 491.371 & LysoPA (22:2/0:0) & 1.25 & 1.14 & 359.313 & MG (18:0/0:0/0:0) & 1.15 & 0.98 \\
\hline 494.329 & LysoPC (16:1) & 1.22 & 1.27 & 381.304 & MG (20:3/0:0/0:0) & 1.03 & 1.02 \\
\hline 544.341 & LysoPC (20:4) & 1.22 & 1.01 & 357.093 & MG (18:1/0:0/0:0) & 0.78 & 1.05 \\
\hline 568.342 & LysoPC (22:6) & 1.11 & 1.03 & 403.234 & MG (22:6/0:0/0:0) & 0.22 & 0.96 \\
\hline 502.299 & LysoPE (20:4) & 1.09 & 0.9 & 355.358 & MG (0:0/18:2/0:0) & 0.15 & 1.08 \\
\hline 454.294 & LysoPE (16:0) & 1.08 & 0.97 & \multicolumn{4}{|c|}{ Diacyl glycerols } \\
\hline 518.325 & LysoPC (18:3) & 0.98 & 0.98 & 643.283 & DG (18:0/20:5/0:0) & 1.96 & 0.59 \\
\hline 496.342 & LysoPC (16:0) & 0.9 & 0.91 & 591.15 & DG (16:0/18:3/0:0) & 1.6 & 1.14 \\
\hline 542.323 & LysoPC (20:5) & 0.88 & 1.04 & 609.161 & DG (18:1/17:0/0:0) & 1.6 & 1.17 \\
\hline 508.359 & LysoPE (20:1) & 0.74 & 0.93 & 631.144 & DG (18:4/19:0/0:0) & 1.23 & 1.09 \\
\hline 545.402 & LysoPI (14:0/0:0) & 0.63 & 1.02 & 561.403 & DG (14:1/18:3/0:0) & 1.07 & 0.93 \\
\hline 452.392 & LysoPE (16:1) & 0.51 & 0.92 & 607.251 & DG (18:2/17:0/0:0) & 1.01 & 1.03 \\
\hline 478.32 & LysoPE (18:2) & 0.44 & 0.52 & 617.259 & DG (18:2/18:2/0:0) & 0.96 & 1.16 \\
\hline 480.424 & Lyso PC (O-16:1/0:0) & 0.43 & 1.42 & 663.457 & DG (20:4/20:5/0:0) & 0.91 & 0.99 \\
\hline \multicolumn{4}{|c|}{ Glycerophospholipids } & 599.247 & DG (18:4/17:2/0:0) & 0.85 & 1.07 \\
\hline 780.553 & $\mathrm{PC}(36: 5)[\mathrm{M}+\mathrm{H}]$ & 2.05 & 1.29 & 601.264 & DG (18:3/17:2/0:0) & 0.83 & 1.09 \\
\hline 758.568 & $\mathrm{PC}(34: 2)[\mathrm{M}+\mathrm{H}]$ & 1.09 & 1.01 & 615.244 & DG (18:2/18:3/0:0) & 0.77 & 1.11 \\
\hline 816.59 & PC (38:2) [M + H] & 0.99 & 1.08 & 603.22 & DG (18:2/17:2/0:0) & 0.66 & 1.2 \\
\hline 744.585 & PE (36:2) & 0.96 & 1.17 & 589.428 & DG (16:1/18:3/0:0) & 0.62 & 1.01 \\
\hline 784.583 & $\mathrm{PC}(36: 3)[\mathrm{M}+\mathrm{H}]$ & 0.95 & 1.06 & 513.415 & DG (12:0/16:0/0:0) & 0.56 & 1.04 \\
\hline 760.582 & PC (34:1) [M + H] & 0.92 & 0.98 & 635.143 & DG (18:2/19:0/0:0) & 0.48 & 0.94 \\
\hline 734.569 & PC (32:0) [M + H] & 0.90 & 1.1 & 633.147 & DG (18:3/19:0/0:0) & 0.46 & 0.9 \\
\hline
\end{tabular}


Table 2. Cont.

\begin{tabular}{|c|c|c|c|c|c|c|c|}
\hline $\mathrm{m} / \mathrm{z}$ & & $\mathrm{HC} / \mathrm{C}$ & $\mathrm{HCd} / \mathrm{HCs}$ & $\mathrm{m} / \mathbf{z}$ & & $\mathrm{HC} / \mathrm{C}$ & $\mathrm{HCd} / \mathrm{HCs}$ \\
\hline \multicolumn{4}{|c|}{ Steroids, Incl.Bile Acids } & \multicolumn{4}{|c|}{ Fatty Acid Derivatives } \\
\hline 810.596 & $\mathrm{PC}(36: 1)[\mathrm{M}+\mathrm{Na}]$ & 0.83 & 1.41 & 579.294 & DG (16:1/17:1/0:0) & 0.37 & 0.94 \\
\hline 808.582 & $\mathrm{PC}(36: 2)[\mathrm{M}+\mathrm{Na}]$ & 0.80 & 1.24 & 665.582 & DG (20:3/20:5/0:0) & 0.07 & 1.36 \\
\hline 633.254 & PA (O-16:0/16:1) & 0.79 & 1.08 & \multicolumn{4}{|c|}{ Phosphoglycerols } \\
\hline 786.602 & $\mathrm{PC}(36: 2)[\mathrm{M}+\mathrm{H}]$ & 0.78 & 1.02 & 509.322 & PG (18:2/0:0) & 1.32 & 0.78 \\
\hline 806.568 & $\mathrm{PC}(36: 3)[\mathrm{M}+\mathrm{Na}]$ & 0.76 & 0.93 & 483.347 & PG (16:1/0:0) & 0.88 & 0.81 \\
\hline 782.564 & $\mathrm{PC}(36: 4)[\mathrm{M}+\mathrm{H}]$ & 0.69 & 1.14 & 485.376 & PG (16:0/0:0) & 0.48 & 1.2 \\
\hline 804.55 & $\mathrm{PC}(36: 4)[\mathrm{M}+\mathrm{Na}]$ & 0.24 & 1 & 709.164 & PG (O-16:0/16:0) & 0.26 & 0.85 \\
\hline 704.21 & PC (30:1) & 0.24 & 0.83 & 707.167 & PG (O-16:0/16:1) & 0.26 & 0.84 \\
\hline 702.213 & PC (30:2) & 0.23 & 0.83 & & & & \\
\hline
\end{tabular}

\subsubsection{Discrimination Analysis: PCA and PLSDA for HC vs. C Groups}

First, the unsupervised PCA was conducted, showing an explained co-variance of $40.6 \%$ for the first two components in the HC vs. C groups (data not shown). The discrimination between the HC and C groups was better represented by PLSDA (with a co-variance of $40.1 \%$ ) (Figure 1a) and the VIP scores derived from the PLSDA loadings showed the first 15 molecules to be considered as putative biomarkers of discrimination between the two groups (Figure 1b). The cross-validation algorithm showed high accuracy (close to one), a high R2 and significantly high Q2 values $>0.95$. These data indicated good predictability for this model.

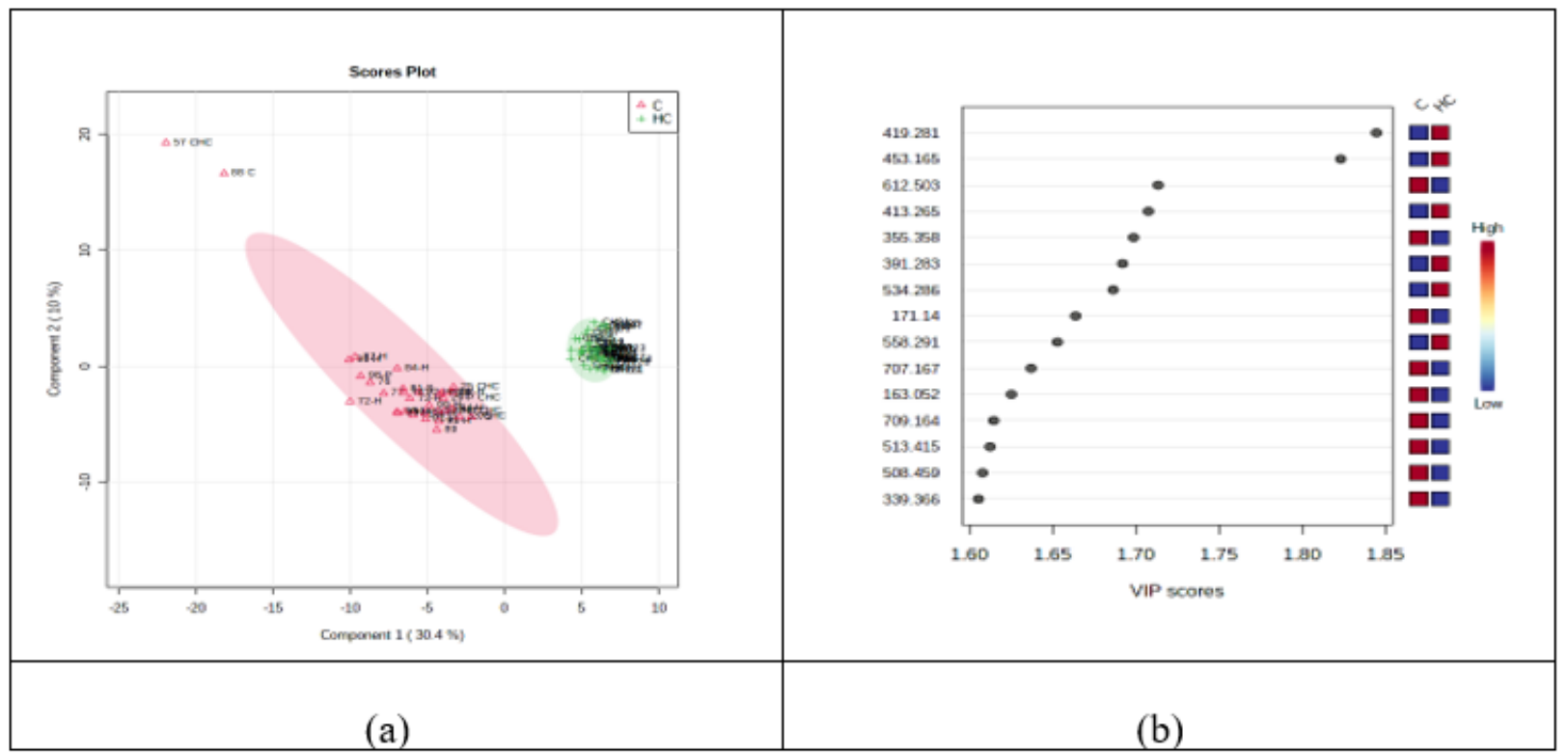

Figure 1. (a) PLSDA plot with samples' identification, showing the discrimination between $C$ and HC groups. (b) VIP scores derived from PLSDA loadings.

Based on the PLSDA loadings graphic and VIP scores, there were noticeably increased levels of six molecules in the HC group: 1,25-dihydroxy cholesterol $(\mathrm{m} / \mathrm{z}=419.281)$, myristyl palmitate $(\mathrm{m} / \mathrm{z}=453.165)$, 25-hydroxy vitamin D2 $(\mathrm{m} / \mathrm{z}=413.265), 12$-ketodeoxycholic acid $(\mathrm{m} / \mathrm{z}=391.283)$, lysoPC (21:4) $(\mathrm{m} / \mathrm{z}=558.291)$, and lysoPE $(22: 2)(\mathrm{m} / \mathrm{z}=534.286)$. Meanwhile, decreases in the HC group were observed for ceramide (d18:0/20:0 (2OH)) $(\mathrm{m} / \mathrm{z}=612.503), M G(0: 0 / 18: 2 / 0: 0)(\mathrm{m} / \mathrm{z}=355.358)$, decenoic acid $\mathrm{C} 10(\mathrm{~m} / \mathrm{z}=171.140)$, hydroxy adipic acid $(\mathrm{m} / \mathrm{z}=163.052), \mathrm{PG}(\mathrm{O}-16: 0 / 16: 0)(\mathrm{m} / \mathrm{z}=709.164), \mathrm{DG}(12: 0 / 16: 0 / 0: 0)$ 
( $\mathrm{m} / \mathrm{z}=513.415)$, lysoPE $(20: 1)(\mathrm{m} / \mathrm{z}=508.459)$, and dimethyl-2-eicosenoic acid $(\mathrm{m} / \mathrm{z}=339.366)$.

Table 3 represents the data released from the $t$-test and fold-change analysis, showing the tendency of molecules to evolve between $\mathrm{HC}$ and $\mathrm{C}$ groups.

Table 3. The $\mathrm{m} / \mathrm{z}$ values and identification of molecules with the most significant differences between the HC and C groups, based on the $p$ values (from $t$-tests), fold-change, and Log2FC showing the tendency of evolution in the HC vs. C groups. I-Increase; D-decrease.

\begin{tabular}{|c|c|c|c|c|c|}
\hline $\mathrm{m} / \mathrm{z}$ & Identification & $p$ Value & FC & $\log 2 \mathrm{FC}$ & Tendency HC vs. C \\
\hline 419.281 & 1,25-dihydroxy cholesterol & $5.94 \times 10^{-26}$ & 5.98 & 2.5805 & I \\
\hline 453.165 & Myristyl palmitate & $1.45 \times 10^{-24}$ & 3.35 & 1.7482 & I \\
\hline 391.283 & 12-keto deoxycholic acid & $2.36 \times 10^{-18}$ & 2.61 & 1.385 & $\mathrm{I}$ \\
\hline 534.286 & lysoPE (22:2) & $3.82 \times 10^{-18}$ & 2.03 & 1.1245 & I \\
\hline 558.291 & LysoPC (21:4) & $5.62 \times 10^{-17}$ & 2.17 & 1.1177 & $\mathrm{I}$ \\
\hline 413.265 & 25-hydroxy vitamin D2 & $6.06 \times 10^{-19}$ & 2.48 & 1.3153 & I \\
\hline 355.358 & MG (18:2/0:0/0:0) & $1.34 \times 10^{-18}$ & 0.12 & -3.0437 & $\mathrm{D}$ \\
\hline 612.503 & Ceramide (d18:0/20:0 (2OH)) & $3.56 \times 10^{-19}$ & 0.28 & -1.8195 & D \\
\hline 171.140 & Decenoic acid (C10:0) & $2.40 \times 10^{-17}$ & 0.04 & -4.4131 & $\mathrm{D}$ \\
\hline 707.167 & PG (O-16:0/16:1) & $1.83 \times 10^{-16}$ & 0.20 & -2.3198 & $\mathrm{D}$ \\
\hline 163.052 & Hydroxy adipic acid & $4.27 \times 10^{-16}$ & 0.21 & -2.22 & $\mathrm{D}$ \\
\hline 709.164 & PG (O-16:0/16:0) & $8.98 \times 10^{-16}$ & 0.20 & -2.3214 & $\mathrm{D}$ \\
\hline 513.415 & PG (18:0/0:0) & $1.04 \times 10^{-15}$ & 0.44 & -1.1718 & $\mathrm{D}$ \\
\hline 508.459 & Ceramide (d18:2/14:0) \& isom & $1.42 \times 10^{-15}$ & 0.37 & -1.4338 & $\mathrm{D}$ \\
\hline 339.366 & $\begin{array}{l}\text { Dimethyl eicosanoic acid } \\
\text { (C20:1) }\end{array}$ & $1.67 \times 10^{-15}$ & 0.13 & -2.8875 & $\mathrm{D}$ \\
\hline 415.209 & Ascorbyl palmitate & $3.74 \times 10^{-13}$ & 2.50 & 1.3232 & $\mathrm{D}$ \\
\hline 702.213 & PC (30:2) & $1.31 \times 10^{-14}$ & 0.17 & -2.5291 & $\mathrm{D}$ \\
\hline 704.210 & PC $(30: 1)$ & $6.58 \times 10^{-15}$ & 0.18 & -2.4645 & $\mathrm{D}$ \\
\hline 596.512 & Cer (d18:0/20:0) and isom & $2.41 \times 10^{-13}$ & 0.37 & -1.3964 & $\mathrm{D}$ \\
\hline 524.448 & Ceramide (d18:1/15:0) & $7.40 \times 10^{-13}$ & 0.30 & -1.6999 & $\mathrm{D}$ \\
\hline 568.459 & Ceramide (d18:0/18:0) & $1.37 \times 10^{-12}$ & 0.35 & -1.4968 & $\mathrm{D}$ \\
\hline 529.407 & Linoleyl linoleate \& isomers & $1.72 \times 10^{-14}$ & 0.33 & -1.5771 & $\mathrm{D}$ \\
\hline 496.419 & Ceramide (d15:1/16:0) & $1.04 \times 10^{-12}$ & 0.41 & -1.2777 & $\mathrm{D}$ \\
\hline 149.024 & Methionine & $2.63 \times 10^{-13}$ & 0.21 & -2.2327 & $\mathrm{D}$ \\
\hline 249.189 & C16:4 fatty acid & $2.46 \times 10^{-15}$ & 0.18 & -2.4734 & $\mathrm{D}$ \\
\hline 257.204 & Palmitic acid C16:0 & $4.62 \times 10^{-12}$ & 0.15 & -2.7155 & $\mathrm{D}$ \\
\hline 383.207 & 16,16-dimethyl-PGE1 & $4.46 \times 10^{-4}$ & 0.15 & -2.6836 & $\mathrm{D}$ \\
\hline
\end{tabular}

3.2.3. Random Forest Analysis, Heatmaps and Biomarker Analysis for HC vs. C Groups

Figure $2 a, b$ represent the graphics of random forest analysis, and heatmaps showing the illustration of differences between the samples and groups. 


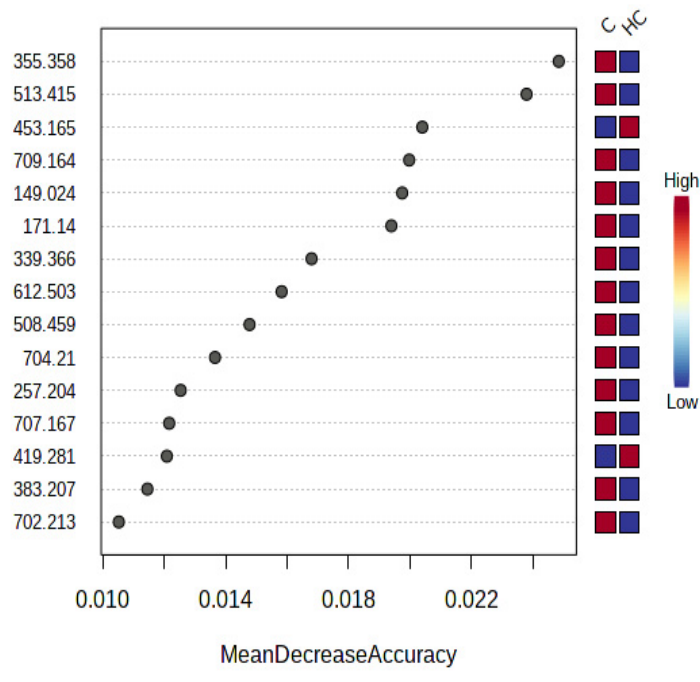

(a)

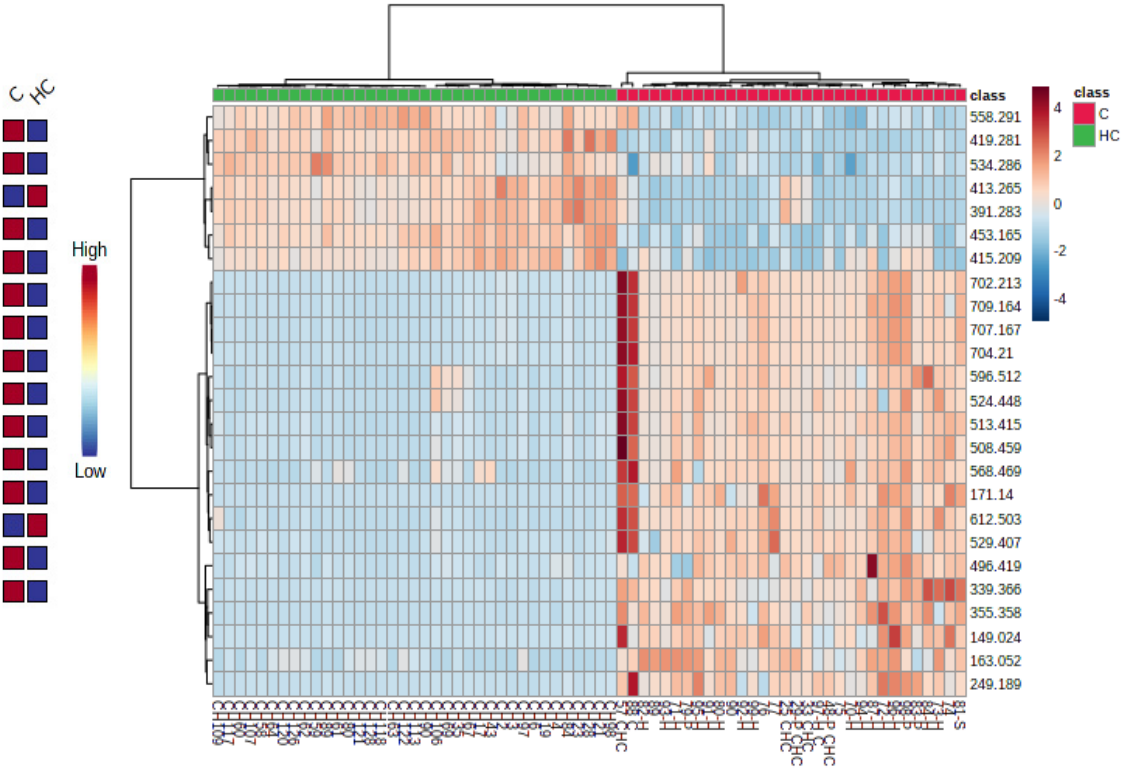

(b)

Figure 2. (a) Random forest graph showing the mean decrease in accuracy for the molecules as putative biomarkers of differentiation between the HC and C groups. (b) Heatmap showing the clusters and molecules responsible for the differentiation between the $\mathrm{HC}$ and $\mathrm{C}$ group.

Biomarker analysis allowed the calculation of sensitivity versus specificity for each molecule, represented by the AUC values in the ROC curves. Table 4 shows the $\mathrm{m} / \mathrm{z}$ values and molecule identifications, the AUC values higher than 0.989 , the Log2FC and $p$-values of each molecule considered as a potential biomarker, and their variation in the HC vs. C groups. Simultaneously, Figure 3 illustrates the AUROC values for the six most representative metabolites in our study.

Table 4. The $\mathrm{m} / \mathrm{z}$ values and molecules with an AUC $>0.989$, where the most significant $p$-values show the variations between the HC and C groups. The value and sign of the Log2FC score show the decrease (D) or increase (I) of molecule variation (negative values are associated with increases in molecules in the HC group, and positive values with decreases in the HC group). I-increase; D-decrease.

\begin{tabular}{ccccc}
\hline $\mathbf{m} / \mathbf{z}$ & Identification & $\begin{array}{c}\boldsymbol{p} \text { Value } \\
(\boldsymbol{t} \text {-Tests) }\end{array}$ & Log2 FC & Variation (HC vs. C) \\
\hline 419.281 & 1,25 dihydroxy Cholesterol & $1.5071 \times 10^{-33}$ & -2.138 & $\mathrm{I}$ \\
453.165 & Myristyl palmitate & $1.4840 \times 10^{-29}$ & -2.041 & $\mathrm{I}$ \\
558.291 & LysoPC (21:4) & $9.5558 \times 10^{-24}$ & -1.501 & $\mathrm{I}$ \\
534.286 & LysoPE (22:2) & $7.8846 \times 10^{-18}$ & -1.056 & $\mathrm{I}$ \\
513.415 & PG (18:0/0:0) & $5.1513 \times 10^{-28}$ & 0.838 & $\mathrm{D}$ \\
508.459 & Ceramide (d18:2/14:0) \& isom & $1.2346 \times 10^{-32}$ & 1.092 & $\mathrm{D}$ \\
633.147 & DG (18:3/19:0/0:0)[iso2] & $5.7123 \times 10^{-13}$ & 1.113 & $\mathrm{D}$ \\
612.503 & Ceramide (d18:0/20:0 (2OH)) & $2.3868 \times 10^{-28}$ & 1.481 & $\mathrm{D}$ \\
709.164 & PG (O-16:0/16:0) & $2.9093 \times 10^{-34}$ & 1.953 & $\mathrm{D}$ \\
707.167 & PG (O-16:0/16:1) & $6.0675 \times 10^{-40}$ & 1.953 & $\mathrm{D}$ \\
704.21 & PC (30:1) & $2.3366 \times 10^{-43}$ & 2.079 & $\mathrm{D}$ \\
702.213 & PC (30:2) & $2.4668 \times 10^{-36}$ & 2.142 & $\mathrm{D}$ \\
339.366 & Dimethyl eicosanoic acid (C20:1) & $9.6275 \times 10^{-15}$ & 2.602 & $\mathrm{D}$ \\
355.358 & MG (0:0/18:2/0:0) & $8.10591 \times 10^{-19}$ & 2.750 & $\mathrm{D}$ \\
171.14 & Decenoic acid (C10:0) & $5.9068 \times 10^{-22}$ & 4.051 & $\mathrm{D}$ \\
\hline
\end{tabular}



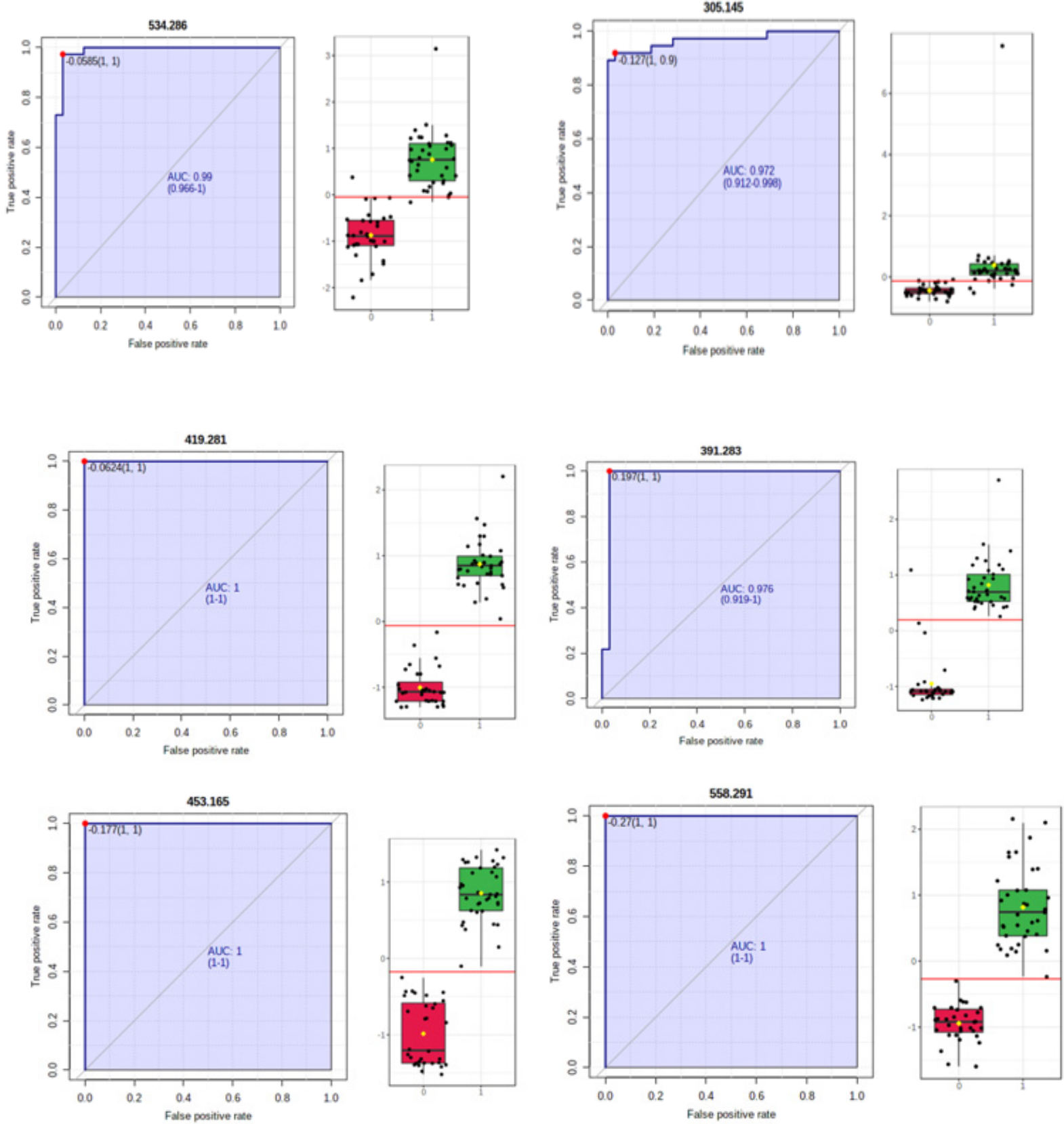

Figure 3. ROC curves of the most significant metabolites: 1,25-dihydroxy cholesterol $(\mathrm{m} / \mathrm{z}=419.281)$, 12-ketodeoxycholic acid $(\mathrm{m} / \mathrm{z}=391.283)$, myristyl palmitate $(\mathrm{m} / \mathrm{z}=453.165)$, lysoPC (21:4) $(\mathrm{m} / \mathrm{z}=558.291)$, lysoPE $(22: 2)(\mathrm{m} / \mathrm{z}=534.286)$, and arachidonic acid $(\mathrm{m} / \mathrm{z}=305.145)$.

\subsubsection{Correlations between Lipid Metabolites, and Clinical and Biological Characteristics}

The relation of different lipid metabolites and clinical and biological parameters was assessed using Spearman correlations. For each HCC patient referred for surgery, portal hypertension was measured by hepatic venous portal flow gradient (HVPG). Moreover, it has been established that values above $10 \mathrm{mmHg}$, equivalent to clinically significant portal hypertension, are associated with post hepatectomy liver failure [19]. Since HVPG measurement is not widely available and is considered an invasive procedure, the pursuit of finding biomarkers to identify patients at risk is paramount. Using Spearman correlations, we have found out that, in HCC patients who underwent surgery, C16 sphingosine tends to predict clinically significant portal hypertension $(p=0.06, \mathrm{R}=-0.05)$, and sphingosine- 1 phosphate correlates with HVPG. 
A total of 22 out of 37 HCC patients (59.45\%) presented tumor recurrence at five year follow-up, after the initial diagnosis and treatment. Dismally, no metabolite could definitively predict tumor relapse. Since C16-sphingosine tends to correlate, and sphingosine-1 phosphate correlates, with HVPG, we can extrapolate and attest that C16-sphingosine and sphingosine-1 phosphate are associated with recurrence and post-hepatectomy liver failure. More mathematical models are needed which include metabolites to determine the patients at risk.

We have pointed out that 1,25-dihydroxy cholesterol $(\mathrm{m} / \mathrm{z}=419.281)$, myristyl palmitate $(\mathrm{m} / \mathrm{z}=453.165), 12-$ ketodeoxycholic acid $(\mathrm{m} / \mathrm{z}=391.283)$, lysoPC $(21: 4)(\mathrm{m} / \mathrm{z}=558.291)$, and lysoPE $(22: 2)(\mathrm{m} / \mathrm{z}=534.286)$ are putative biomarkers that differentiate HCC from cirrhosis. Along these lines, we have investigated whether these biomarkers have a role in decompensation and prognosis. The deterioration of liver function in a patient with cirrhosis is characterized by jaundice, ascites, hepatic encephalopathy, hepatorenal syndrome, or variceal bleeding. In total, among the included patients, eight developed ascites, three developed variceal bleeding, and one hepatic encephalopathy. In a univariate analysis among the HVPG $(\mathrm{OR}=0.82,95 \% \mathrm{CI}: 0.8-1.24, p<0.005)$ and MELD score $(\mathrm{OR}=0.74$, 95\% CI: 0.54-0.93), arachidonic acid was the only metabolite to predict decompensation $(\mathrm{OR}=0.1,95 \% \mathrm{CI}: 0-0.16, p<0.005)$. In the multivariate analysis, unfortunately, no variable was associated with decompensation.

\subsubsection{Univariate Analysis to Predict the Death (Comparison of HCd vs. HCs Groups)}

To identify which patients were at risk, Cox regression analysis was applied to further evaluate the predictive capacity of critical metabolites and assess the overall survival of HCC patients. During a follow-up period of five years, 13 (35.13\%) out of 37 patients died, and, among them, 8 had developed a decompensation episode. The survival of patients with all lipid parameters as single variables was examined, using the Cox proportional hazard method for death. The highest HR for death was found for myristyl palmitate $(\mathrm{HR}=16.67), p=0.005$ (Table 3$)$. Furthermore, as adjusting variables in the multivariate Cox regression models, MELD and HVPG were added as predictors for survival. Adjusting for MELD score and HVPG in a multivariate analysis (Table 5) we have found that arachidonic acid ( $\mathrm{HR}=12.08,95 \% \mathrm{CI}$ : 2.95-49.93, $p<0.005), 1$,25-dihydroxy cholesterol (HR $=10.61,95 \%$ CI: 1.37-82.3, $p<0.005)$, myristyl palmitate $(\mathrm{HR}=31.63,95 \% \mathrm{CI}$ : 1.51-661.66, $p<0.005), 12-k e t o$ deoxycholic acid (HR $=21.98,95 \%$ CI: $1.39-121.39, p<0.005)$, lysoPC (21:4) $(\mathrm{HR}=10.46,95 \% \mathrm{CI}: 1.41-77.54, p<0.005)$, and lysoPE $(22: 2)(\mathrm{HR}=2.37,95 \%$ CI: $1.07-5.24, p<0.005)$, are independent markers of survival.

Table 5. Unadjusted hazard ratio with Cox proportional hazard method for death in lipid HCC metabolism and multivariate analysis of lipid components when adjusted to MELD and HVPG.

\begin{tabular}{ccccccc}
\hline & \multicolumn{3}{c}{ Univariate Analysis } & \multicolumn{3}{c}{ Multivariate Analysis } \\
\hline Parameter & HR & $\mathbf{9 5 \%}$ CI & $\boldsymbol{p}$ & HR & $\mathbf{9 5 \%} \mathbf{C I}$ & $\boldsymbol{p}$ \\
\hline Arachidonic acid & 1.56 & $(0.52-4.69)$ & 0.429 & 12.08 & $(2.95-49.93)$ & $\mathbf{0 . 0 2 7}$ \\
1,25 dihydroxy Cholesterol & 5.29 & $(1.86-15.08)$ & $\mathbf{0 . 0 0 2}$ & 10.61 & $(1.37-82.3)$ & $\mathbf{0 . 0 2 4}$ \\
Myristyl palmitate & 16.67 & $(2.3-120.69)$ & $\mathbf{0 . 0 0 5}$ & 31.63 & $(1.51-661.66)$ & $\mathbf{0 . 0 2 6}$ \\
12-keto deoxycholic acid & 4.96 & $(1.45-17)$ & $\mathbf{0 . 0 1 1}$ & 12.98 & $(1.39-121.39)$ & $\mathbf{0 . 0 2 5}$ \\
LysoPC (21:4) & 4.86 & $(1.81-13.01)$ & $\mathbf{0 . 0 0 2}$ & 10.46 & $(1.41-77.54)$ & $\mathbf{0 . 0 2 2}$ \\
LysoPE (22:2) & 1.54 & $(1.16-2.03)$ & $\mathbf{0 . 0 0 2}$ & 2.37 & $(1.07-5.24)$ & $\mathbf{0 . 0 3 2}$ \\
\hline
\end{tabular}

MELD = model for end-stage liver disease; $\mathrm{HVPG}=$ hepatic venous pressure gradient; lysoPC = lysophosphatidylcholine; lysoPE = lysophosphatidylethanolamine.

\subsubsection{Multivariate Analysis for HCd vs. HC Groups}

Figure 4 shows the PLSDA plot, which reflects the discrimination between $\mathrm{HCd}$ and HCs (marked HC) groups (a), and the molecules with higher VIP scores derived from PLSDA loadings. 


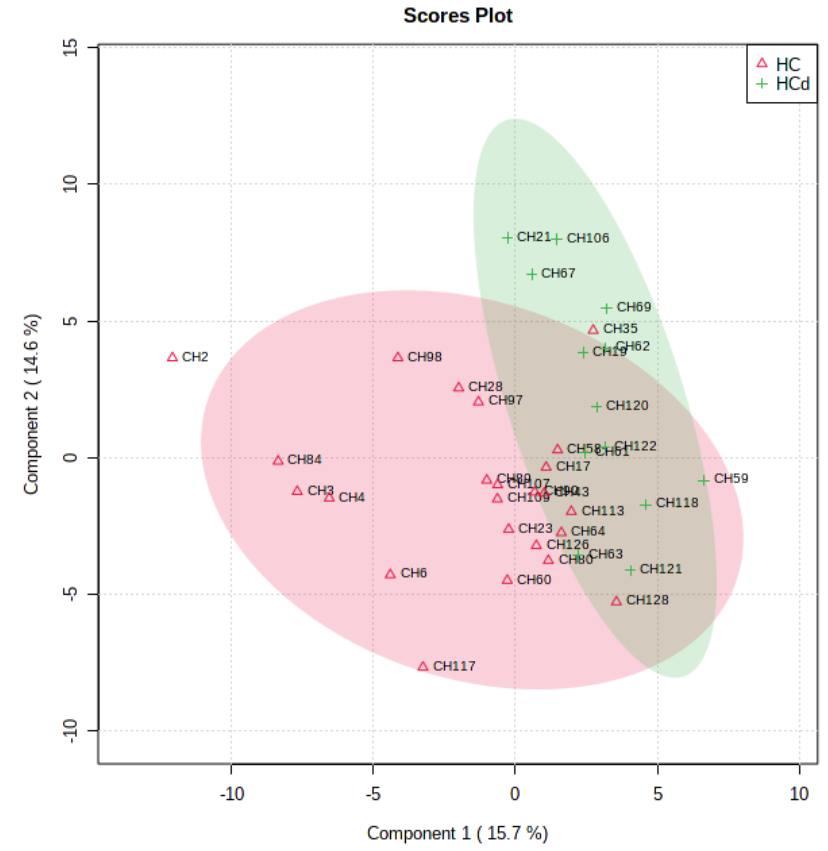

(a)

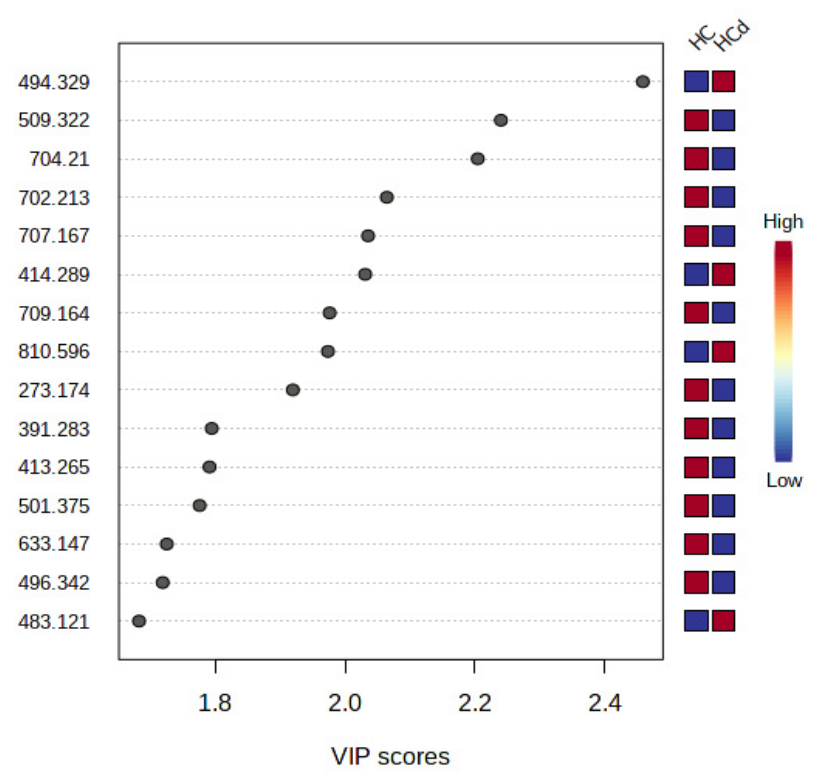

(b)

Figure 4. (a) PLSDA plot with samples' identification, showing the discrimination between the HCd and HCs groups. (b) VIP scores derived from PLSDA loadings.

Figure 5 represents the graphics of random forest analysis (a), and heatmaps showing the illustration of differences between the groups HCd and HCs.

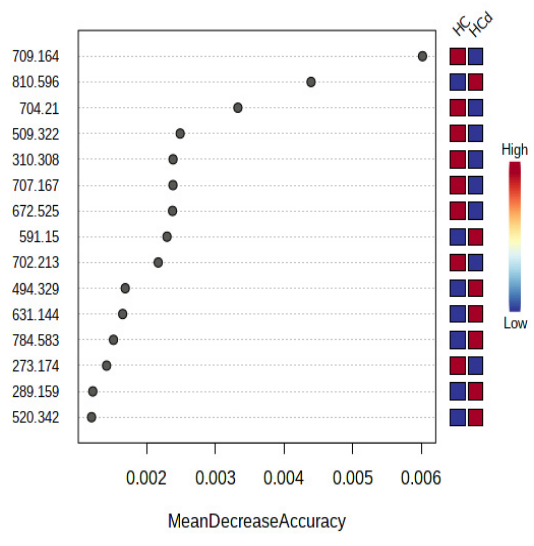

(a)

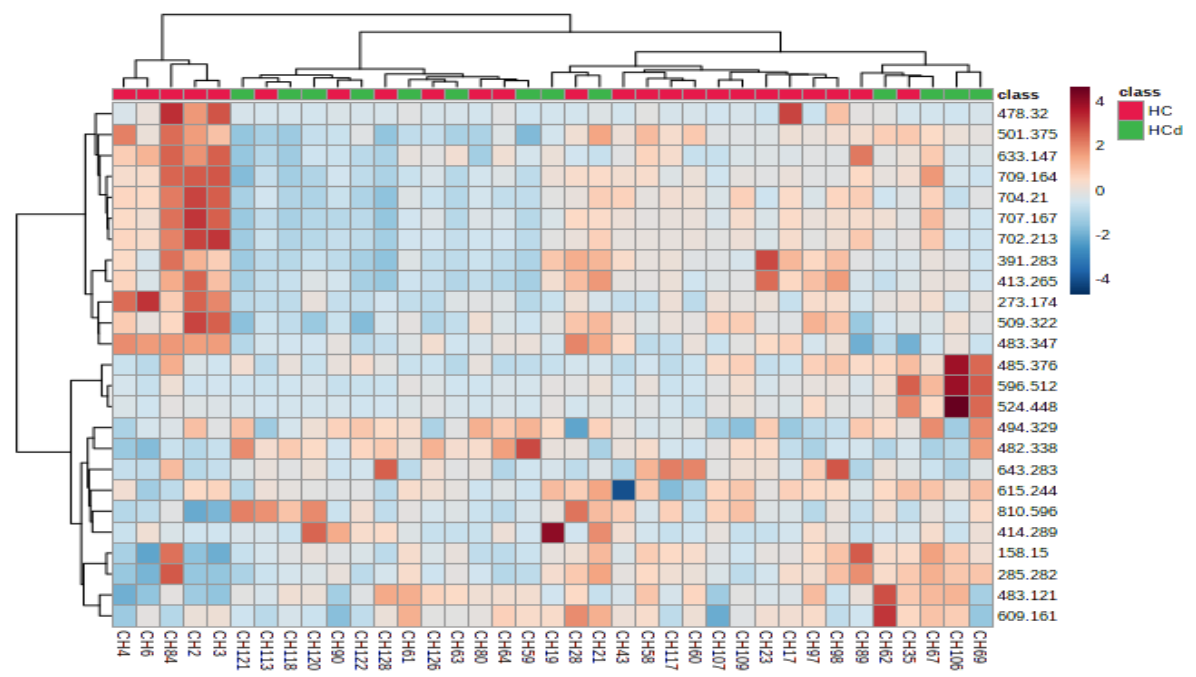

(b)

Figure 5. (a) Random forest graph showing the mean decrease in accuracy for the molecules as putative biomarkers of differentiation between the HCd and HCs groups. (b) Heatmap showing the clusters and molecules responsible for the differentiation between the HCd and HCs groups.

Table 6 shows the first 20 molecules with VIP scores higher than 1.5 and MDA values higher than 0.01, and the AUC values. 
Table 6. Molecules with VIP scores $>1.5$ (see Figure 3) and MDA values $>0.01$ (see Figure 4).

\begin{tabular}{|c|c|c|c|c|c|c|c|c|c|}
\hline $\mathbf{m} / \mathbf{z}$ & Identification & AUC & $p$-Value & HCd vs. HC & $\mathbf{m} / \mathbf{z}$ & Identification & AUC & $p$-Value & HCd vs. HC \\
\hline 702.213 & PC (30:2) & 0.820 & 0.027 & $\mathrm{D}$ & 391.283 & 12-Ketodeoxycholic acid & 0.714 & 0.064 & $\mathrm{D}$ \\
\hline 704.210 & PC $(30: 1)$ & 0.807 & 0.017 & $\mathrm{D}$ & 413.265 & 25-hydroxy vitamin D2 & 0.692 & 0.072 & $\mathrm{D}$ \\
\hline 707.167 & PG $(\mathrm{O}-16: 0 / 16: 1)$ & 0.804 & 0.025 & $\mathrm{D}$ & 273.174 & Estradiol & 0.692 & 0.061 & $\mathrm{D}$ \\
\hline 709.164 & PG (O-16:0/16:0) & 0.791 & 0.025 & $\mathrm{D}$ & 483.121 & PG (16:1/0:0) & 0.684 & 0.064 & I \\
\hline 509.322 & PG (18:2/0:0) & 0.772 & 0.017 & $\mathrm{D}$ & 501.375 & Palmitoleyl linolenate & 0.682 & 0.062 & $\overline{\mathrm{D}}$ \\
\hline 810.596 & PC $(36: 1)$ & 0.762 & 0.034 & I & 633.254 & PA $(O-16: 0 / 16: 1)$ & 0.639 & 0.085 & $\mathrm{D}$ \\
\hline 494.329 & LysoPC (16:1) & 0.761 & 0.017 & I & 591.150 & DG (16:0/18:3/0:0) & 0.637 & 0.127 & I \\
\hline 414.289 & Heptadecanoyl carnitine & 0.642 & 0.046 & I & 496.342 & LysoPC (16:0) & 0.634 & 0.139 & $\mathrm{D}$ \\
\hline 672.525 & $\begin{array}{c}\text { GlycoCeramide } \\
\text { (d18:1/14:0) }\end{array}$ & 0.631 & 0.160 & $\mathrm{D}$ & 631.144 & DG (18:4/19:0/0:0) & 0.625 & 0.240 & I \\
\hline 784.583 & PC (36:3) & $<0.600$ & $>0.05$ & I & 310.308 & Decatrienoyl carnitine & $<0.600$ & $>0.05$ & $\mathrm{D}$ \\
\hline
\end{tabular}

The data obtained highlights that arachidonic acid, 1,25-dihydroxy cholesterol myristyl palmitate, 12-ketodeoxycholic acid, lysoPC (21:4), and lysoPE (22:2) may predict HCC mortality and recurrence, and constitute important biomarkers. Moreover, 12-ketodeoxycholic acid levels seem to decrease for HCC patients at risk of death, and thus might represent an important parameter.

\section{Discussion}

The early detection of hepatocellular carcinoma and the identification of prognostic factors are key to personalized medicine to improve patient outcomes. Along these lines, the main objective of our study was to identify new biomarkers for diagnosing early hepatocellular carcinoma and to identify which patients are at risk of developing tumor recurrence, or even death. Therefore, we conducted an untargeted metabolomics study emphasizing lipid metabolites in 69 European patients-32 compensated cirrhotic patients without HCC (controls) and 37 compensated cirrhotic patients with early HCC (cases) that underwent curative treatment (surgery or ablation), and have followed up with these patients for almost four years.

The AFP values did not show any differences between HCC and cirrhosis patients, most likely due to both groups' compensated underlying liver disease. Therefore, AFP is an imperfect surveillance tool making it imperative for the hepatology and oncology community to find a reasonably suitable hepatic cancer biomarker. Ultra-high-performance liquid chromatography coupled with electrospray ionization quadrupole time-of-flight mass spectrometry (UHPLC-ESI+-QTOF-MS) revealed important serum biomarkers that differentiate HCC patients from cirrhotic controls. The present study has identified different lipid molecules involved in fatty acid, glycerophospholipid, sphingolipid, and acylglycerol metabolism as putative biomarkers for differentiating cirrhosis and HCC, with AUC values over 0.900. Moreover, for the first time, we have identified metabolites that differentiate the deceased HCC patient from those who survived. Hence, we report two types of phosphocholines (PC (30:2); PC (30:1)) with AUROCs of 0.820 and 0.807 , respectively, that are increased in early HCC, with levels that drop when tumors progress. Indeed, several studies have reported changes in gene expression and enzyme activity that led to altered PC synthesis in cancer, which contributes to tumorigenesis, while other studies have revealed oscillating PC expression in HCC patients [20,21]. To our knowledge, the present study contrasts and compares the expression of PC metabolites for the first time between early HCC patients who survived curative treatment and those who died. An emphasis on PC metabolism in the future might bring new treatment options in oncology.

Based on the PLSDA loadings graphics and VIP scores, our study identified several metabolites increased in the HCC group compared to the cirrhotic controls, depicted in Figure 5. The lipid metabolites with the most increased expression were attributed to 1,25-dihydroxy cholesterol, 25-hydroxy vitamin D, myristyl palmitate, 12-ketodeoxycholic acid, lysoPC (21:4), and lysoPE (22:2). Cholesterol plays an intricate role in liver tumorigen- 
esis and supports the growth of hepatocarcinoma lesions depleted of fatty acid synthase in mice and humans [22]. Moreover, it seems that HMG-CoA reductase, a cornerstone enzyme of cholesterol synthesis and the target of statins, is upregulated in human HCC samples and retrospective studies suggest that the use of statins might be associated with a reduced risk of HCC development [23]. Our study found out that 1,25-dihydroxy cholesterol is upregulated by almost six times relative to cirrhotic controls.

Progressing into the lipid maze, the expression of 25-hydroxy vitamin D is a matter of debate. Undoubtedly, low levels of vitamin D are associated with increased mortality in chronic liver disease. In contrast, its increased expression in HCC serum in some studies has been demonstrated to have an antitumoral role, and, in other studies, was associated with tumor progression [24-26]. Bile acids, phosphatidyl-choline, and phosphatidylethanolamine metabolites also play a dual role in cancer biology $[17,20,27]$. Although our study determines minuscule pieces from the puzzle of lipid biology, 1,25-dihydroxy cholesterol, myristyl palmitate, 12-keto deoxycholic acid, lysoPC (21:4), and lysoPE (22:2) represent important independent markers of survival in multivariate analysis, when adjusting for MELD score and portosystemic gradient. Thus, the next step for our study would be to determine a mathematical model encompassing the metabolites mentioned above, and to apply it to other cohorts of cirrhosis and liver cancer to determine which patients are at risk.

Figure 6 represents the general variations in specific molecules between cirrhosis versus early hepatocellular carcinoma.

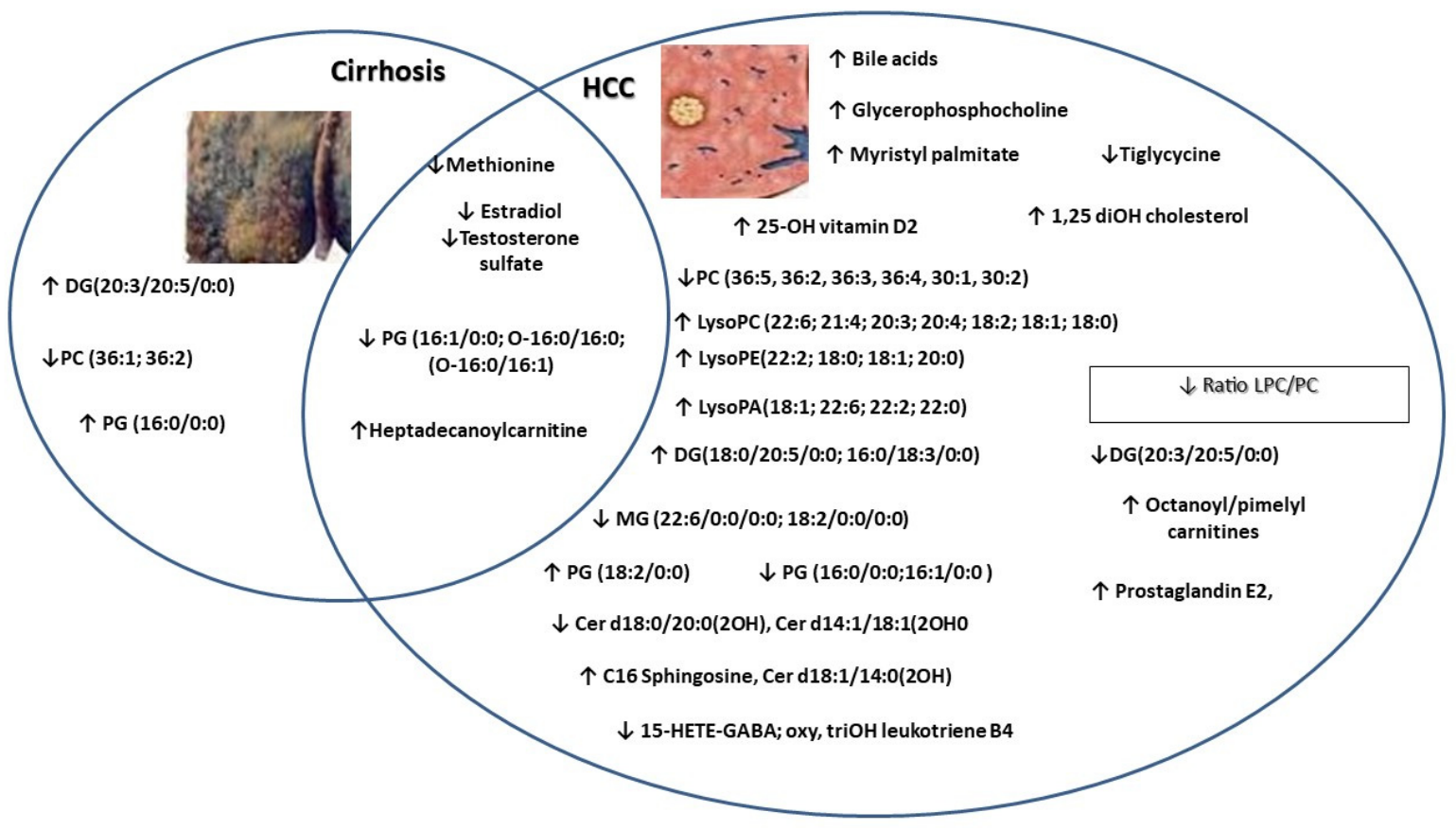

Figure 6. Common and specific molecules which were found to discriminate between compensated cirrhosis versus early hepatocellular carcinoma.

In our study, we have revealed that most ceramides are expressed at lower levels compared to cirrhotic patients. This is most likely due to a depletion of ceramide content during transformation into bioactive molecules with protumor roles. Takashima, Y. and Li, Z. have confirmed these arguments and state that ceramide metabolism is deregulated in primary liver cancer $[28,29]$. Nonetheless, we have conceded that sphingolipid family members discriminate between HCC and cirrhosis, and might represent excellent biomarkers far better than AFP.

Apart from being an essential player in stimulating the apoptosis of tumor cells, chemotherapy and ionizing radiation exert their effects through ceramides, a fact demonstrated by the increased levels of ceramides after exposure to those procedures [30]. There- 
fore, ceramides constitute crucial cancer treatment targets or adjuvant therapies to existing chemotherapies [31]. Since we have obtained increased levels of many ceramides in cirrhosis and decreased levels in HCC patients, we believe that, in future, it would be a cornerstone to determine the cut-off values of ceramides to pinpoint the high-risk moment of developing primary liver cancer. Along these lines, large cohort studies are needed which focus on this direction. Studies attest that S1P is increased in the serum of HCC patients [13]. There is an S1P axis, which refers to all the molecules involved in its metabolism and its receptors or other intracellular targets. S1P is produced (inside the cell) by the phosphorylation of sphingosine, a reaction catalyzed by two sphingosine kinases: SPHK1 and SPHK2. SPHK1 is of great importance between these two kinases, because its increased activity stimulates cell growth and inhibits apoptosis. Once activated inside the cell, S1P can act on intracellular targets or be secreted in the interstitial area and interact with cell surface receptors, a process known as "inside-out" signaling. The importance of this axis lies in the fact that its components can be therapeutic targets in cancer treatment. For example, monoclonal antibodies directed against S1P have been synthesized (sonepcizumab), alongside SPHK1 inhibitors, and various agonists and antagonists of the S1P receptors (S1PRs), and are in several phases of clinical trials [14]. Although we obtained results in contrast with those in the literature, we consider that the low level of S1P obtained in our patients with HCC is due to the presence of compensated underlying liver disease. It is likely that S1P increases in more advanced stages of cirrhosis or tumor progression.

There is scarce data regarding the role of lipids as prognostic markers in HCC. One study from 2019 from a French group revealed elevated concentrations of phosphatidylcholine (PC) 16:0/16:1 ( $p=0.0180)$, PC 16:0/16:0 ( $p=0.0327)$, PC 16:0/18:1 ( $p=0.0264)$ and sphingomyelin (SM) 18:2/24:1 ( $p=0.0379)$, and low concentrations of lyso-phosphatidylcholine 20:4 (0.0093) and plasmalogen-phosphatidyl-ethanolamine (LysoPE) 16:0/20:4 $(p=0.0463)$, LysoPE 18:0/20:4 $(p=0.0077)$, LysoPE 18:0/20:5 $(p=0.0163)$, and LysoPE 18:0/20:3 ( $p=0.0463)$ as good biomarkers for HCC versus cirrhosis, as well as two ceramides (ceramide $\mathrm{d} 18: 1 / 26: 0$ and ceramide $\mathrm{d} 18: 1 / 24: 1$ ) that were associated with the risk of death in one and/or three years [20]. Moreover, in a Chinese cohort, it seems that changes in polyunsaturated-eicosapentaenoic acid, docosahexaenoic acid, and linolenic acid are associated with early tumor recurrence after hepatectomy. In addition, the researchers also found that $85 \%$ of early recurrent HCC s can be predicted with an AUROC equal to 0.95 in a training set with the combination of methionine, GCDCA, and cholesterol sulfate [32]. Another Chinese study identified, after univariate and multivariate Cox regression, the combined retinol and retinal panel as an independent predictor for HCC, and showed that the low expression of the panel was correlated with decreased survival after hepatectomy. Retinol and retinal discriminate HCC from cirrhosis with an AUROC of 0.996 and 0.994 in tissue, and 0.812 and 0.744 in serum, respectively [33]. No metabolite identified in our study could determine tumor relapse. Nevertheless, since C16-sphinganine tends to correlate, and sphingosine-1 phosphate correlates, with HVPG, we can extrapolate and attest that C16-sphinganine and sphingosine-1 phosphate are associated with recurrence and post-hepatectomy liver failure. Arachidonic acid was the only metabolite to predict decompensation. In the multivariate analysis, dismally, no variable was associated with decompensation. When adjusting for MELD score and HVPG in a multivariate analysis, we report for the first time that the increased expression of 1,25-dihydroxy cholesterol, myristyl palmitate, 12-keto deoxycholic acid, lysoPC (21:4), and lysoPE (22:2) are independent markers of survival.

Although most studies emphasize serum metabolites, other authors have performed metabolomics on tissues or other biological fluids as follows. One study from China reported that the retinol metabolic signature determined from liver biopsy and serum had considerable diagnostic and prognostic value for identifying HCC patients who would benefit from prompt therapy. Other authors claim that tissue metabolomics yields a more precise biochemical information pattern when searching for tumoral energy metabolites [33]. 
In the last few years, the concept of prognostic scores has been reported in oncology. Different mathematical models comprising biochemical analyses were released to assess tumor relapse or identify patients at risk of developing complications. Naturally, metabolomics also gained terrain. In 2020, Wang Q et al. reported a global prognostic index (GPI) score for operated HCC patients that combines a metabolite panel with satellite nodes for assessing overall survival. Compared with the current clinical classification systems, including the Barcelona clinic liver cancer (BCLC) stage, the tumor node metastasis (TNM) stage, and the albumin-bilirubin (ALBI) grade, the GPI score presented a notable performance according to the time-dependent receiver operating, and might stand as a helpful tool to stratify the HCC prognostic risk after surgery [34]. Other scientists used gas chromatography-mass spectrometry (GC-MS)-based metabolomics, identifying phenylalanine and galactose and including them in two mathematical models to predict the risk of mortality, recurrence, and metastasis with essential results. Moreover, some reports attest to different discriminatory biomarkers between HCC and cirrhosis when performing metabolomics protocols from urine or feces [35,36].

Our study presents several limitations. The number of patients was too small to conduct a complete characterization of biomarkers. These results need to be confirmed in a more significant number of patients. Additionally, more studies with European cohorts need to be performed, as we have compared our results with Chinese patients and there may be differences. Another limitation of our study is the inclusion of HCC patients with different etiologies of the underlying liver disease. Although the underlying etiology in most cases is chronic viral hepatitis $C$ as in the control group, patients with other etiologies have been added to include a sufficient number of patients. One final limitation of our study is the inclusion of 18 patients at the BCLC B stage. Although these patients are classified as at an intermediate stage, they have a preserved liver function and have undergone curative treatments, such as surgery and percutaneous ablation.

Overall, while many metabolites appear to display excellent diagnostic performances in HCC, reproducibility stands as a significant issue to address. In the future, it will be paramount to include patients with decompensated cirrhosis, and intermediary and advanced HCC to broaden the metabolite spectrum of liver disease. In addition, in the literature, we have seen that validation cohorts have a minimal number of studies, most of them retrospective and with no statistical adjustments for important confounding variables such as smoking status, alcohol consumption, lifestyle habits, physical activity, body mass index, or waist circumference. With this in mind, given the rise of HCC developed on non-alcoholic fatty liver disease, it would be far-reaching to conduct a different study assessing this matter. Lipid metabolism is the metabolic hub of NAFLD with bioactive sphingolipids, as a hallmark of NAFLD and NAFLD-derived HCC [37].

\section{Conclusions}

Due to the high incidence of cancer, and particularly hepatocellular carcinoma, the search for non-invasive biomarkers is crucial in modern medicine. Such markers should be useful in the very early diagnosis of the disease, and in determining the prognosis and monitoring of the disease course and treatment. Moreover, it would be interesting to perform a metabolomic study on patients with hepatic cancer that underwent ablation, trans-arterial chemoembolization, or required systemic treatment to find out which are the best candidates for the treatment, and to monitor the patients appropriately.

In conclusion, our study reveals missing pieces of the lipid puzzle that not only differentiate compensated cirrhosis and early hepatocellular carcinoma, but also identify patients at risk. Specifically, it was determined for the first time that arachidonic acid, myristylpalmitate, and the family members of phosphatidyl-choline are markers of survival, and might constitute future prognostic biomarkers in a personalized and minimally invasive medicine practice. We believe that by navigating the old maze of lipids discovered years ago, we could find answers to current struggles in hepatology and oncology. 
Supplementary Materials: The following supporting information can be downloaded at: https:// www.mdpi.com/article/10.3390/jcm11051292/s1, Table S1: Molecules separated and identified $(\mathrm{n}=154)$ by LC-MS: average peak intensities, standard deviation (SD). The $\mathrm{m} / \mathrm{z}$ values represent $[M+1]$ values where M- molecular mass; Table S2: Molecules separated and identified $(n=154)$ by LC-MS: average peak intensities (Id, I), standard deviations (SDd, SD)s for group HCd $(n=13)$ vs HCs $(n=24)$. The $m / z$ values represent $[M+1]$ values where $M-$ molecular mass.

Author Contributions: Conceptualization, I.N., B.P. and H.S.; methodology I.N., I.M., T.M., C.M. and C.S.; software, D.L.; validation, M.G., Z.S., B.P., G.A.F. and H.S.; formal analysis, M.G., G.A.F. and Z.S.; investigation, I.N., I.M. and T.M.; resources, C.M. and C.S.; data curation, H.S.; writing-original draft preparation, I.N.; writing-review and editing, I.N., I.M., T.M. and C.S.; visualization, Z.S.; supervision, G.A.F., B.P., H.S. and C.S.; project administration, H.S.; funding acquisition, M.G. and H.S. All authors have read and agreed to the published version of the manuscript.

Funding: The results of this research were partially funded by the University of Medicine and Pharmacy "Iuliu Hatieganu" (Ph.D. study Iuliana Nenu-4322/01.10.2018), L'OREAL Women for Science award and UEFISCDI through the PN-III-P1-1.2-PCCDI-2017-0221/59PCCDI/2018 (IMPROVE) project.

Institutional Review Board Statement: This study was conducted according to the guidelines of the Declaration of Helsinki, and approved by the Institutional Review Board (or Ethics Committee) of the Regional Institute of Gastroenterology and Hepatology, O. Fodor.

Informed Consent Statement: Informed consent was obtained from all subjects involved in the study.

Data Availability Statement: Not applicable.

Acknowledgments: “Iuliu Hatieganu” University of Medicine and Pharmacy, ACAMed, POCU—125171.

Conflicts of Interest: The authors declare no conflict of interest.

\section{References}

1. Fitzmaurice, C.; Akinyemiju, T.F.; Al Lami, F.H.; Alam, T.; Alizadeh-Navaei, R.; Allen, C.; Alsharif, U.; Alvis-Guzman, N.; Amini, E.; Anderson, B.O.; et al. Global, regional, and national cancer incidence, mortality, years of life lost, years lived with disability, and disability-adjusted life-years for 29 cancer groups, 1990 to 2016 a systematic analysis for the global burden of disease study global burden o. JAMA Oncol. 2018, 4, 1553-1568. [CrossRef] [PubMed]

2. Akinyemiju, T.; Abera, S.; Ahmed, M.; Alam, N.; Alemayohu, M.A.; Allen, C.; Al-Raddadi, R.; Alvis-Guzman, N.; Amoako, Y.; Artaman, A.; et al. The Burden of Primary Liver Cancer and Underlying Etiologies from 1990 to 2015 at the Global, Regional, and National Level. JAMA Oncol. 2017, 3, 1683-1691. [CrossRef] [PubMed]

3. Cabibbo, G.; Enea, M.; Attanasio, M.; Bruix, J.; Craxí, A.; Cammà, C. A meta-analysis of survival rates of untreated patients in randomized clinical trials of hepatocellular carcinoma. Hepatology 2010, 51, 1274-1283. [CrossRef] [PubMed]

4. Chaiteerakij, R.; Addissie, B.D.; Roberts, L.R. Update on Biomarkers of Hepatocellular Carcinoma. Clin. Gastroenterol. Hepatol. 2015, 13, 237-245. [CrossRef]

5. Mocan, T.; Simão, A.L.; Castro, R.E.; Rodrigues, C.M.P.; Słomka, A.; Wang, B.; Strassburg, C.; Wöhler, A.; Willms, A.G.; Kornek, M. Liquid biopsies in hepatocellular carcinoma: Are we winning? J. Clin. Med. 2020, 9, 1514. [CrossRef]

6. Wang, X.; Zhang, A.; Sun, H. Power of metabolomics in diagnosis and biomarker discovery of hepatocellular carcinoma. Hepatology 2013, 57, 2072-2077. [CrossRef]

7. Spratlin, J.L.; Serkova, N.J.; Eckhardt, S.G. Clinical applications of metabolomics in oncology: A review. Clin. Cancer Res. 2009, 15, 431-440. [CrossRef]

8. Hanahan, D. Hallmarks of Cancer: New Dimensions. Cancer Discov. 2022, 12, 31-46. [CrossRef]

9. Nenu, I.; Gafencu, G.A.; Popescu, T.; Kacso, G. Lactate-A new frontier in the immunology and therapy of prostate cancer. J. Cancer Res. Ther. 2017, 13, 406-411. [CrossRef]

10. Nagarajan, S.R.; Butler, L.M.; Hoy, A.J. The diversity and breadth of cancer cell fatty acid metabolism. Cancer Metab. 2021, 9, 1-28. [CrossRef]

11. Wang, M.; Han, J.; Xing, H.; Zhang, H.; Li, Z.; Liang, L.; Li, C.; Dai, S.; Wu, M.; Shen, F.; et al. Dysregulated fatty acid metabolism in hepatocellular carcinoma. Hepatic Oncol. 2016, 3, 241-251. [CrossRef] [PubMed]

12. Krautbauer, S.; Meier, E.M.; Rein-Fischboeck, L.; Pohl, R.; Weiss, T.S.; Sigruener, A.; Aslanidis, C.; Liebisch, G.; Buechler, C. Ceramide and polyunsaturated phospholipids are strongly reduced in human hepatocellular carcinoma. Biochim. Biophys. Acta-Mol. Cell Biol. Lipids 2016, 1861, 1767-1774. [CrossRef] [PubMed]

13. Pyne, N.J.; Pyne, S. Sphingosine 1-phosphate and cancer. Nat. Rev. Cancer 2010, 10, 489-503. [CrossRef]

14. Kunkel, G.T.; MacEyka, M.; Milstien, S.; Spiegel, S. Targeting the sphingosine-1-phosphate axis in cancer, inflammation and beyond. Nat. Rev. Drug Discov. 2013, 12, 688-702. [CrossRef] [PubMed] 
15. Grammatikos, G.; Schoell, N.; Ferreirós, N.; Bon, D.; Herrmann, E.; Farnik, H.; Köberle, V.; Piiper, A.; Zeuzem, S.; Kronenberger, B.; et al. Serum sphingolipidomic analyses reveal an upregulation of C16-ceramide and sphingosine-1-phosphate in hepatocellular carcinoma. Oncotarget 2016, 7, 18095-18105. [CrossRef]

16. Buechler, C.; Aslanidis, C. Role of lipids in pathophysiology, diagnosis and therapy of hepatocellular carcinoma. Biochim. Biophys. Acta-Mol. Cell Biol. Lipids 2020, 1865, 158658. [CrossRef]

17. Sun, L.; Beggs, K.; Borude, P.; Edwards, G.; Bhushan, B.; Walesky, C.; Roy, N.; Manley, M.W.; Gunewardena, S.; O’Neil, M.; et al. Bile acids promote diethylnitrosamine-induced hepatocellular carcinoma via increased inflammatory signaling. Am. J. Physiol.Gastrointest. Liver Physiol. 2016, 311, G91-G104. [CrossRef]

18. Cucchetti, A.; Cescon, M.; Golfieri, R.; Piscaglia, F.; Renzulli, M.; Neri, F.; Cappelli, A.; Mazzotti, F.; Mosconi, C.; Colecchia, A.; et al. Hepatic venous pressure gradient in the preoperative assessment of patients with resectable hepatocellular carcinoma. J. Hepatol. 2016, 64, 79-86. [CrossRef]

19. Bolondi, L.; Burroughs, A.; Dufour, J.F.; Galle, P.R.; Mazzaferro, V.; Piscaglia, F.; Raoul, J.L.; Sangro, B. Heterogeneity of patients with intermediate (BCLC B) Hepatocellular Carcinoma: Proposal for a subclassification to facilitate treatment decisions. Semin Liver Dis. 2012, 32, 348-359. [CrossRef]

20. Ismail, I.T.; Elfert, A.; Helal, M.; Salama, I.; El-Said, H.; Fiehn, O. Remodeling lipids in the transition from chronic liver disease to hepatocellular carcinoma. Cancers 2021, 13, 88. [CrossRef]

21. Cotte, A.K.; Cottet, V.; Aires, V.; Mouillot, T.; Rizk, M.; Vinault, S.; Binquet, C.; De Barros, J.P.P.; Hillon, P.; Delmas, D. Phospholipid profiles and hepatocellular carcinoma risk and prognosis in cirrhotic patients. Oncotarget 2019, 10, 2161-2172. [CrossRef] [PubMed]

22. Che, L.; Chi, W.; Qiao, Y.; Zhang, J.; Song, X.; Liu, Y.; Li, L.; Jia, J.; Pilo, M.G.; Wang, J.; et al. Cholesterol biosynthesis supports the growth of hepatocarcinoma lesions depleted of fatty acid synthase in mice and humans. Gut 2020, 69, 177-186. [CrossRef] [PubMed]

23. Singh, S.; Singh, P.P.; Singh, A.G.; Murad, M.H.; Sanchez, W. Statins are associated with a reduced risk of hepatocellular cancer: A systematic review and meta-analysis. Gastroenterology 2013, 144, 323-332. [CrossRef] [PubMed]

24. Finkelmeier, F.; Kronenberger, B.; Köberle, V.; Bojunga, J.; Zeuzem, S.; Trojan, J.; Piiper, A.; Waidmann, O. Severe 25hydroxyvitamin D deficiency identifies a poor prognosis in patients with hepatocellular carcinoma-A prospective cohort study. Aliment. Pharmacol. Ther. 2014, 39, 1204-1212. [CrossRef]

25. Liu, H.; Jiang, X.; Qiao, Q.; Chen, L.; Matsuda, K.; Jiang, G.; Yu, T.; Wang, Y.; Lin, H.; Liang, X.; et al. Association of circulating 25-Hydroxyvitamin D and its related genetic variations with hepatocellular carcinoma incidence and survival. Ann. Transl. Med. 2020, 8, 1080. [CrossRef] [PubMed]

26. Lai, G.Y.; Wang, J.B.; Weinstein, S.J.; Parisi, D.; Horst, R.L.; McGlynn, K.A.; Männistö, S.; Albanes, D.; Freedman, N.D. Association of 25-Hydroxyvitamin D with Liver Cancer Incidence and Chronic Liver Disease Mortality in Finnish Male Smokers of the ATBC Study. Cancer Epidemiol. Biomarkers Prev. 2018, 27, 1075-1082. [CrossRef] [PubMed]

27. Guan, Y.; Chen, X.; Wu, M.; Zhu, W.; Arslan, A.; Takeda, S.; Nguyen, M.H.; Majeti, R.; Thomas, D.; Zheng, M.; et al. The phosphatidylethanolamine biosynthesis pathway provides a new target for cancer chemotherapy. J. Hepatol. 2020, 72, 746-760. [CrossRef]

28. Takashima, Y.; Horisawa, K.; Udono, M.; Ohkawa, Y.; Suzuki, A. Prolonged inhibition of hepatocellular carcinoma cell proliferation by combinatorial expression of defined transcription factors. Cancer Sci. 2018, 109, 3543-3553. [CrossRef]

29. Li, Z.; Guan, M.; Lin, Y.; Cui, X.; Zhang, Y.; Zhao, Z.; Zhu, J. Aberrant lipid metabolism in hepatocellular carcinoma revealed by liver lipidomics. Int. J. Mol. Sci. 2017, 18, 2550. [CrossRef]

30. Fisher-Wellman, K.H.; Hagen, J.T.; Neufer, P.D.; Kassai, M.; Cabot, M.C. On the nature of ceramide-mitochondria interactions -Dissection using comprehensive mitochondrial phenotyping. Cell. Signal. 2021, 78, 109838. [CrossRef]

31. Morad, S.A.F.; Cabot, M.C. Ceramide-orchestrated signalling in cancer cells. Nat. Rev. Cancer 2013, 13, 51-65. [CrossRef] [PubMed]

32. Zhou, L.; Liao, Y.; Yin, P.; Zeng, Z.; Li, J.; Lu, X.; Zheng, L.; Xu, G. Metabolic profiling study of early and late recurrence of hepatocellular carcinoma based on liquid chromatography-mass spectrometry. J. Chromatogr. B Anal. Technol. Biomed. Life Sci. 2014, 966, 163-170. [CrossRef] [PubMed]

33. Han, J.; Han, M.; Xing, H.; Li, Z.; Yuan, D.; Wu, H.; Zhang, H.; Wang, M.; Li, C.; Liang, L.; et al. Tissue and serum metabolomic phenotyping for diagnosis and prognosis of hepatocellular carcinoma. Int. J. Cancer 2020, 146, 1741-1753. [CrossRef] [PubMed]

34. Wang, Q.; Su, B.; Dong, L.; Jiang, T.; Tan, Y.; Lu, X.; Liu, X.; Lin, X.; Xu, G. Liquid Chromatography-Mass Spectrometry-Based Nontargeted Metabolomics Predicts Prognosis of Hepatocellular Carcinoma after Curative Resection. J. Proteome Res. 2020, 19, 3533-3541. [CrossRef]

35. Osman, D.; Ali, O.; Obada, M.; El-Mezayen, H.; El-Said, H. Chromatographic determination of some biomarkers of liver cirrhosis and hepatocellular carcinoma in Egyptian patients. Biomed. Chromatogr. 2017, 31, e3893. [CrossRef]

36. Cao, H.; Huang, H.; Xu, W.; Chen, D.; Yu, J.; Li, J.; Li, L. Fecal metabolome profiling of liver cirrhosis and hepatocellular carcinoma patients by ultra performance liquid chromatography-mass spectrometry. Anal. Chim. Acta 2011,691, 68-75. [CrossRef]

37. Simon, J.; Ouro, A.; Ala-Ibanibo, L.; Presa, N.; Delgado, T.C.; Martínez-Chantar, M.L. Sphingolipids in non-alcoholic fatty liver disease and hepatocellular carcinoma: Ceramide turnover. Int. J. Mol. Sci. 2020, 21, 40. [CrossRef] 